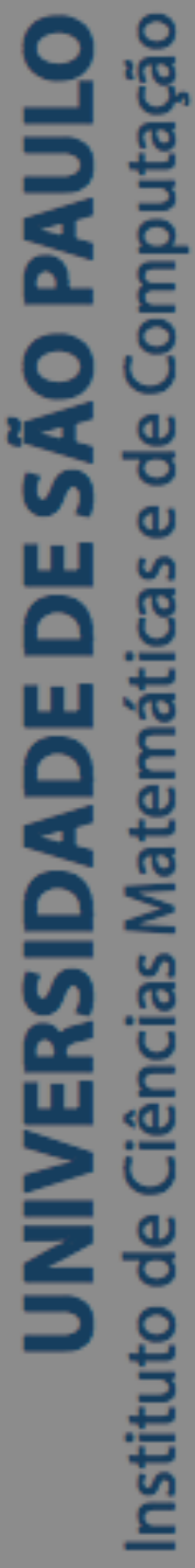

\title{
Processo de Bernoulli correlacionado
}

\section{Ricardo De Carli Novaes}

Dissertação de Mestrado do Programa Interinstitucional de Pós-Graduação em Estatística (PIPGEs) 

Data de Depósito:

Assinatura:

\section{Ricardo De Carli Novaes}

\section{Processo de Bernoulli correlacionado}

\begin{abstract}
Dissertação apresentada ao Departamento de Estatística - DEs/UFSCar e ao Instituto de Ciências Matemáticas e de Computação - ICMC-USP, como parte dos requisitos para obtenção do título de Mestre em Estatística - Programa Interinstitucional de Pós-Graduação em Estatística UFSCar-USP. VERSÃO REVISADA
\end{abstract}

Orientador: Prof. Dr. Renato Jacob Gava

\section{São Carlos}

Agosto de 2019 
Ficha catalográfica elaborada pela Biblioteca Prof. Achille Bassi e Seção Técnica de Informática, ICMC/USP, com os dados fornecidos pelo(a) autor (a)

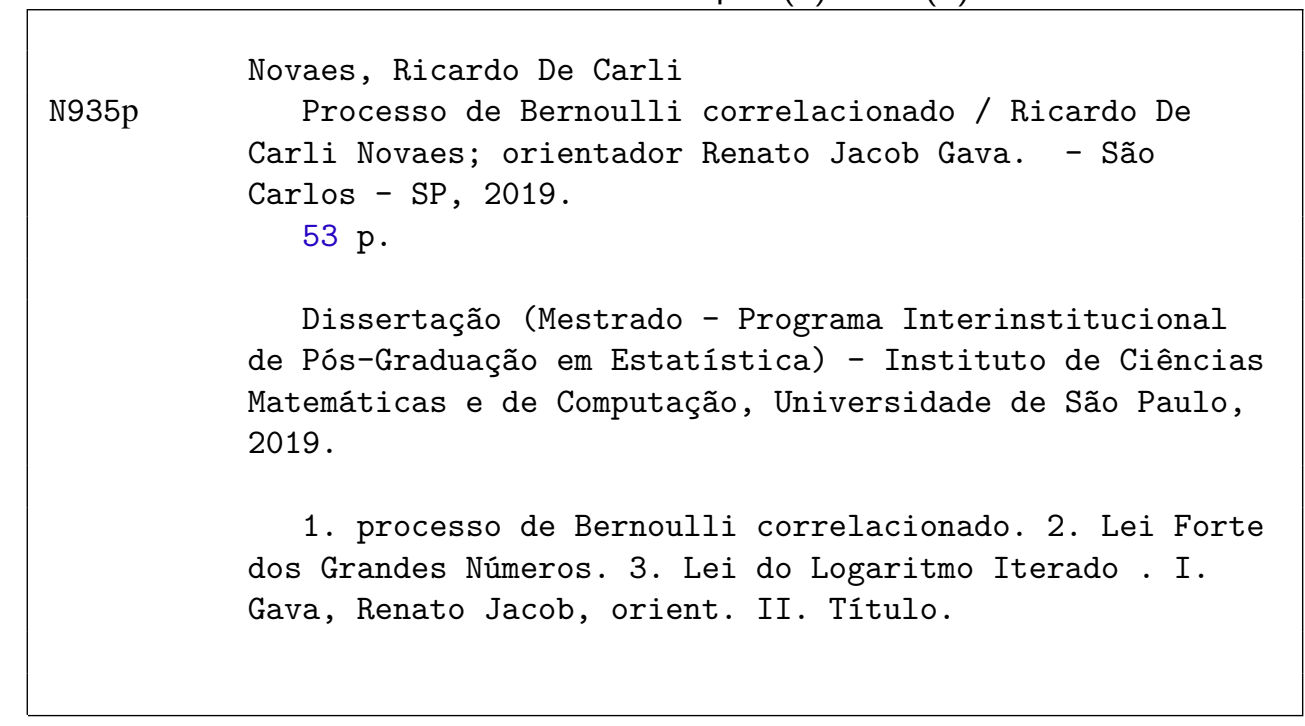




\title{
Ricardo De Carli Novaes
}

\section{Correlated Bernoulli Process}

\begin{abstract}
Master dissertation submitted to the Institute of Mathematics and Computer Sciences - ICMC-USP and to the Department of Statistics - DEs-UFSCar, in partial fulfillment of the requirements for the degree of the Master Program in Programa Interinstitucional de Pós Graduação em Estatística.
\end{abstract}

FINAL VERSION

Advisor: Prof. Dr. Renato Jacob Gava

\section{USP/UFSCar - São Carlos}

Aug 2019 



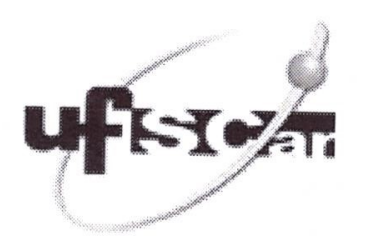

\section{UNIVERSIDADE FEDERAL DE SÃO CARLOS}

Centro de Ciências Exatas e de Tecnologia

Programa Interinstitucional de Pós-Graduação em Estatística

\section{Folha de Aprovação} Assinaturas dos membros da comissão examinadora que avaliou e aprovou a Defesa de Dissertação de Mestrado do
candidato Ricardo de Carli Novaes, realizada em 28/06/2019:
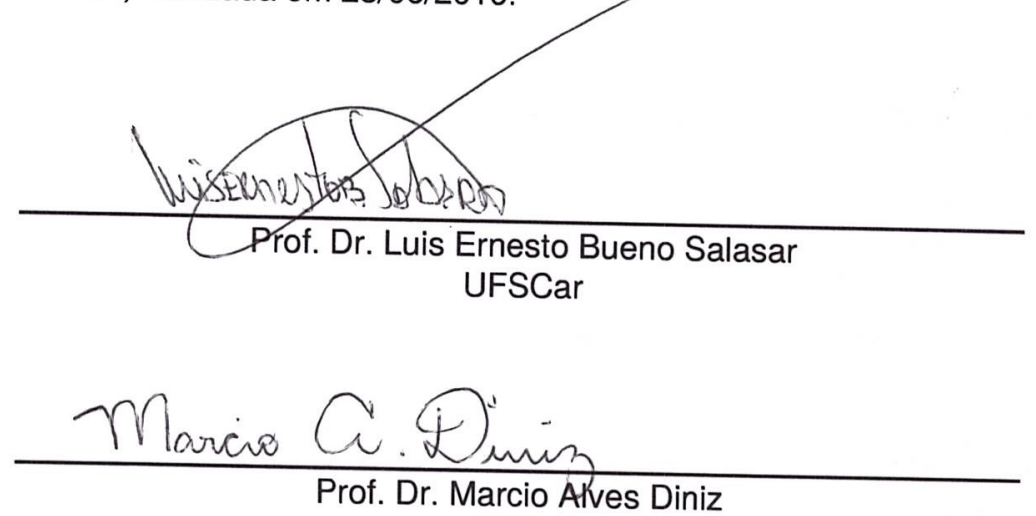

Prof. Dr. Marcio Alves Diniz

UFSCar

Colett; Cuction Fovio

Prof. Dr. Cristian Favio Coletti

UFABC 

Dedico este trabalho aos futuros alunos do programa. Que nunca desistam dos seus objetivos independente de suas dificuldades. 

Agradeço a minha família, que sempre me apoiou nos momentos mais difíceis, principalmente ao meu pai, Marco Aurélio de Castro Novaes, e à minha mãe, Susette Leanira de Carli Novaes.

Agradeço também à Marília Thomé Nadalini, que sempre me incentivou a seguir com os estudos. Por fim, agradeço à USP-ICMC, à Universidade Federal de São Carlos e à Capes pela oportunidade de aprimorar meus conhecimentos em estatística e contribuir pelo desenvolvimento de futuros profissionais. 

"Some things take so long, but how do I explain when not too many people can see we're all the same and because of all their tears, their eyes can't hope to see the beauty that surrounds them" 



\section{RESUMO}

NOVAES, R. C.. Processo de Bernoulli correlacionado. 2019. 53 f. Dissertação (Mestrado em em Estatística - Programa Interinstitucional de Pós-Graduação em Estatística) - Instituto de Ciências Matemáticas e de Computação (ICMC/USP), São Carlos - SP.

O processo de Bernoulli independente, que nada mais é que uma sequência de variáveis aleatórias indenpendentes com distribuição Bernoulli, já é amplamente conhecido na literatura estatística. Esta dissertação lida com uma generalização de tal processo: o processo de Bernoulli correlacionado, isto é, variáveis aleatórias Bernoulli dependentes em que a probabilidade de sucesso num determinado instante $n+1$ é uma função linear do número de sucessos até o instante n. Para este modelo, apresentamos a Lei Forte dos Grandes Números, o Teorema Central do Limite e a Lei do Logaritmo Iterado.

Palavras-chave: processo de Bernoulli correlacionado, Lei Forte dos Grandes Números, Lei do Logaritmo Iterado . 



\section{ABSTRACT}

NOVAES, R. C.. Processo de Bernoulli correlacionado. 2019. 53 f. Dissertação (Mestrado em em Estatística - Programa Interinstitucional de Pós-Graduação em Estatística) - Instituto de Ciências Matemáticas e de Computação (ICMC/USP), São Carlos - SP.

The independent Bernoulli process, which is a sequence of independent Bernoulli random variables, is already widely known in the statistical literature. This masters thesis works with a generalization of this process: the correlated Bernoulli process, that is, dependent Bernoulli random variables in which the probabilityof success at time $n+1$ is a linear function of the number of successes until time $n$. For this model, we present the Strong Law of Large Numbers, the Central Limit Theorem and Law of the Iterated Logarithm.

Key-words: correlated Bernoulli process, Strong Law of the Large Numbers, Central Limit Theorem, Law of the Iterated Logarithm. 



\section{LISTA DE ILUSTRAÇÕES}

Figura 1 - Implicações entre tipos de convergência. . . . . . . . . . . . . . . . . . . . . . . . .

Figura $2-$ GBD aplicado no beisebol. . . . . . . . . . . . 33 

INTRODUÇÃO $\ldots \ldots \ldots \ldots \ldots \ldots \ldots$

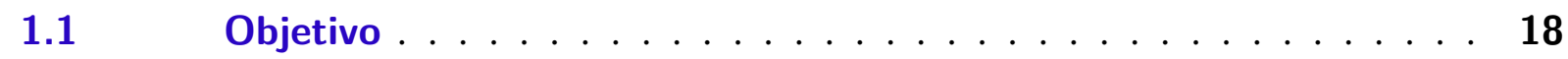

2 CONCEITOS DE CONVERGÊNCIA E MARTINGALES . . . . . . 19

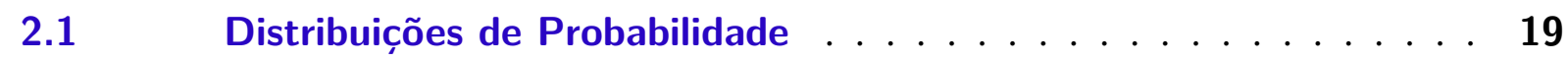

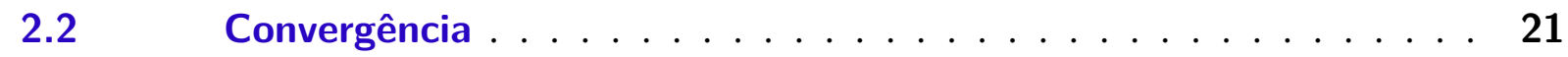

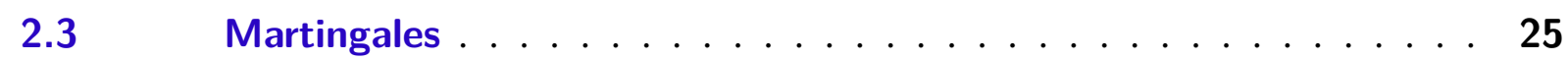

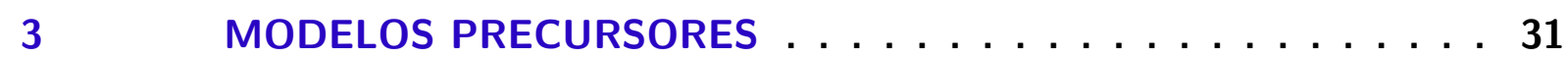

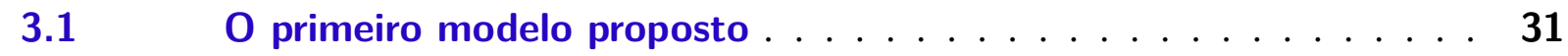

3.1.1 Resultados de Drezner \& Farnum . . . . . . . . . . . . . . 32

3.1.2 Resultados de Heyde . . . . . . . . . . . . . . . . . . 33

$4 \quad$ RESULTADOS DE JAMES, JAMES E QI $2008 \ldots \ldots \ldots$

$4.1 \quad$ Modelo proposto por James, James e Qi (2008) . . . . . . . . 35

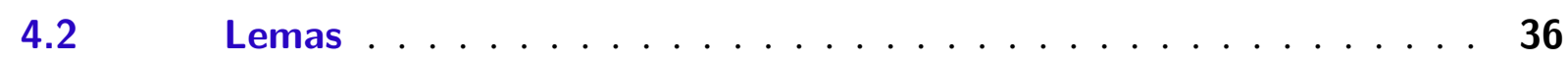

$4.3 \quad$ Lei Forte dos Grandes Números . . . . . . . . . . . . . . . 40

$4.4 \quad$ Teorema Central do Limite . . . . . . . . . . . . . . . . . 42

$4.5 \quad$ Reciprocidade da Lei Forte dos Grandes Números . . . . . . . . . 44

$4.6 \quad$ Lei do Logaritmo Iterado . . . . . . . . . . . . . . . . . . 44

$5 \quad$ CONSIDERAÇÕES FINAIS $\ldots \ldots \ldots \ldots \ldots \ldots$

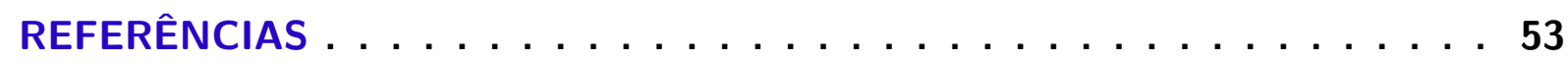





\section{1}

\section{INTRODUÇÃO}

As Variáveis dicotômicas correspondem a experimentos em que só há duas respostas possíveis, como por exemplo sim/não, doente/saudável, morto/vivo, etc. Usualmente escolhemos a característica de interesse e a denominamos de "sucesso"e, consequentemente, chamamos a característica restante de "fracasso". Além disso, atribuimos ao "sucesso"o valor 1 , e ao "fracasso"o valor 0.

O estudo sobre variáveis dicotômicas em probabilidade é comumente associado a variáveis independentes, isto é, o número de sucessos até determinado tempo $n$ não depende dos resultados até o instante $n-1$. Tal suposição de independência, no entanto, é muito questionável.

Tome um exemplo na área de esportes; suponha que em um campeonato de tênis desejemos estudar a probabilidade do jogador A ganhar o $n$-ésimo jogo e defina $X_{n}$ como uma variável aleatória que assume valor 1 quando o jogador A ganha a $n$-ésima partida e 0 quando perde. Claramente, não seria intuitivo assumir que o histórico de sucessos de um jogador não afetará sua partida futura, pois um jogador que vem de derrotas subsequentes provavelmente terá mais chance de perder quando comparado a um jogador que está passando por diversas vitórias seguidas.

$\mathrm{Na}$ teoria das probabilidades denominamos uma sequência (finita ou infinita) de váriaveis aleatórias que assumem apenas valores 0 ou 1 de processo de Bernoulli. Diversos problemas podem ser observados com um processo de Bernoulli, como a sequência de resultados de um jogador de tênis que tomamos como exemplo.

Tem-se, então, uma grande necessidade de generalizar o processo de Bernoulli de modo que a suposição de independência seja enfraquecida. Drezner e Farnum (1993) iniciou o estudo sobre o processo de Bernoulli correlacionado no artigo 'A generalized binomial distribution', em que um primeiro modelo foi proposto. As propriedades assintóticas deste modelo foram estudadas posteriormente no artigo 'Asymptotics and criticality for a correlated Bernoulli process' por Heyde (2004). 
Em um artigo mais recente, denominado 'Limit theorems for correlated Bernoulli random variables', James, James e Qi (2008) propuseram uma generalização do modelo utilizado por Drezner e Farnum, assim como também demonstraram suas propriedades assintóticas.

\subsection{Objetivo}

Esta dissertação tem como objetivo explorar o artigo de James, James e Qi (2008), explicando minuciosamente suas passagens e técnicas, além de proporcionar ao leitor uma revisão sobre o tema e uma breve introdução à convergência e teoria dos martingales.

Para que tal objetivo seja cumprido, os pontos são tratados com a seguinte disposição:

No capítulo 2 é apresentada uma revisão de conceitos de convergência e martingales, trazendo diversas definições e teoremas relevantes para o problema. Em seguida, no decorrer do terceiro capítulo, é feita uma revisão dos modelos precursores apresentados por Drezner e Farnum (1993) e por Heyde (2004).

No capítulo 4 São apresentados os principais resultados de James, James e Qi (2008) e suas respectivas demonstrações de maneira detalhada e comentada. Por fim, no quinto capítulo, os comentários e considerações finais sobre a dissertação são discorridos. 


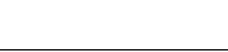

\section{CONCEITOS DE CONVERGÊNCIA E MARTINGALES}

Neste capítulo serão apresentados algumas distribuições de probabilidade, conceitos e resultados de convergência e martingales presentes na literatura. Utilizaremos o livro Introduction to Probability Models de Ross (2010) como referência da primeira seção. Os livros 'Probability and random processes' de Grimmett e Stirzaker (2001), Introduction to Probability Models de Ross (2010) e algumas notas de aula de Jagannathan (2015. Notas de aula) como principais referências para a revisão de convergência. Já para uma revisão sobre teoria de martingales serão utilizados os livros 'Martingale limit theory and its application' de Hall e Heyde (1980) e o livro 'Probability with Martingales' de Williams (1991).

\subsection{Distribuições de Probabilidade}

Nesta seção, serão matematicamente apresentadas as distribuições de probabilidade Bernoulli, Binomial e Normal que se fazem necessárias para o desenvolvimento do texto.

Comecemos pela distribuição Bernoulli:

Definição 1. Suponha um ensaio, ou um experimento, cujo resultado possa ser classificado como "sucesso"ou "fracasso". Se definirmos $X$ como 1 se obtivermos um sucesso e como 0 se obtivermos um fracasso, então a função massa de probabilidade de $X$ é dada por:

$$
p(0)=\mathbb{P}(X=0)=1-p, p(1)=\mathbb{P}(X=1)=p
$$

em que $p, 0 \leq p \leq 1$, é a probabilidade do ensaio ser um "sucesso".

Em suma, dizemos que uma variável aleatória $X$ tem distribuição Bernoulli se sua função massa de probabilidade é dada por (2.1) para algum $p \in(0,1)$. 
Seguimos então para a definição da distribuição Binomial:

Definição 2. Suponha que $n$ ensaios de Bernoulli independentes, cada um com resultados "sucesso"com probabilidade $p$ ou "fracasso"com probabilidade $1-p$ (ensaios de Bernoulli), sejam performados. Se $X$ representa o número de sucessos que ocorreram nas $n$ tentativas, então dizemos que $X$ tem uma distribuição de probabilidade Binomial com parâmetros $(n, p)$.

Sua função de probabilidade é dada por:

$$
p(i)=\left(\begin{array}{c}
n \\
i
\end{array}\right) p^{i}(1-p)^{n-i}, i=0,1, \ldots, n
$$

em que

$$
\left(\begin{array}{c}
n \\
i
\end{array}\right)=\frac{n !}{(n-i) ! i !}
$$

é igual ao número de diferentes grupos de $i$ sucessos que podem ser obtidos em $n$ tentativas.

Segue um exemplo para ilustrar a distribuição Binomial.

Exemplo 1. Quatro moedas são lançadas. Assumindo que os resultados do lançamento de cada moeda são independentes e identicamente distribuídos, qual a probabilidade de termos duas caras e duas coroas?

Sendo $X$ o número de caras obtidas, então $X$ tem distribuição Binomial com parâmetros $(4, p)$. Portanto, por (2.2):

$$
p(2)=\left(\begin{array}{l}
4 \\
2
\end{array}\right) p^{2}(1-p)^{4-2} .
$$

Assumindo que as moedas são justas, isto é $p=\frac{1}{2}$, temos:

$$
\begin{aligned}
p(2) & =\left(\begin{array}{c}
4 \\
2
\end{array}\right) \frac{1}{2}^{2}\left(1-\frac{1}{2}\right)^{4-2} \\
& =\frac{4 !}{(4-2) ! 2 !} \frac{1^{2}}{2} \frac{1^{2}}{2} \\
& =6 \frac{1}{4} \frac{1}{4} \\
& =\frac{3}{8}
\end{aligned}
$$

Por fim, apresentamos a Distribuição de probabilidade Normal, que será utilizada no decorrer da dissertação:

Definição 3. Dizemos que $X$ é uma variável aleatória Normal com parâmetros $\mu \in \mathbb{R}$ e $\sigma^{2} \in \mathbb{R}^{+}$ se a função densidade de probabilidade de $X$ é dada por

$$
f(x)=\frac{1}{\sqrt{2 \pi} \sigma} e^{\frac{-(x-\mu)^{2}}{\sigma^{2}}}, x \in \mathbb{R}
$$


Esta função de probabilidade é conhecida pela curva em formato de sino ao redor da média $\mu$.

\subsection{Convergência}

Nesta seção, serão apresentadas definições de convergência com o intuito de aproximar o leitor dos conceitos necessários para o entendimento da dissertaçāo.

Um dos conceitos mais importantes quando falamos em probabilidade é o de convergência de variáveis aleatórias, que está relacionado aos mais importantes teoremas da estatística, como o Teorema Central do Limite e a Lei Forte dos Grandes Números, que serão apresentados neste capítulo.

Começaremos relembrando qual é a definição de convergência quando tratamos de sequências reais determinísticas.

Definição 4. Seja $\left\{x_{n}\right\}_{n \geq 1}$ uma sequência de valores reais. Dizemos que $x_{n}$ converge para algum valor $x \in \mathbb{R}$ se existe um $n_{0} \in \mathbb{N}$ tal que, para todo $\varepsilon>0$,

$$
\left|x_{n}-x\right|<\varepsilon, \forall n \geq n_{0} \text {. }
$$

Segue um exemplo de convergência de sequências reais:

Exemplo 2. Mostraremos que a sequência $\left\{\frac{1}{n}\right\}_{n \geq 1}$, converge para zero. Tome $n_{0}(\varepsilon)=\left\lceil\frac{1}{\varepsilon}\right\rceil$ (o menor inteiro maior que $\frac{1}{\varepsilon}$ ).

Note que $\left|\frac{1}{n}-0\right|<\varepsilon, \forall n \geq n_{0}(\varepsilon)$. Isto é, $\frac{1}{n}$ converge para 0 .

Agora serão apresentadas diversas notações de convergência para sequências de variáveis aleatórias. Seja $(\Omega, \mathscr{F}, \mathbb{P})$ o espaço de probabilidade e seja $\left\{X_{n}\right\}_{n \in \mathbb{N}}$ uma sequência de variáveis aleatórias de valores reais definidas neste espaço de probabilidade.

Definição 5. Uma sequência de variáveis aleatórias $\left\{X_{n}\right\}_{n \in \mathbb{N}}$ converge certamente (ponto a ponto) para $X$ se

$$
X_{n}(\omega) \rightarrow X(\omega) \quad \forall \omega \in \Omega
$$

Note que para todo $\omega \in \Omega,\left\{X_{n}(\omega)\right\}_{n \in \mathbb{N}}$ é uma sequência de números reais e, portanto, a convergência para esta sequência é a mesma apresentada na Definição 4.

Definição 6. Dizemos que uma sequência de variáveis aleatórias $\left\{X_{n}\right\}_{n \in \mathbb{N}}$ converge quase certamente ou com probabilidade 1 (q.c. ou c.p. 1) para $X$ se

$$
\mathbb{P}\left(\left\{\omega \in \Omega \mid X_{n}(\omega) \rightarrow X(\omega)\right\}\right)=1 .
$$


A convergência quase certa exige que o conjunto dos $\omega \in \Omega$ em que a variável aleatória convirja tenha probabilidade 1 , o que permite que em um conjunto de medida nula, a variável aleatória não convirja. Esta definição de convergência tem suposições enfraquecidas quando comparada a convergência ponto a ponto, mas muitas vezes as suposições para convergência quase certa são muito restritivas. Portanto, outras definições de convergências são apresentadas.

Definição 7. Dizemos que uma sequência de variáveis aleatórias $\left\{X_{n}\right\}_{n \in \mathbb{N}}$ converge em probabilidade (e.p.) para $X$ se

$$
\lim _{n \rightarrow \infty} \mathbb{P}\left(\left|X_{n}-X\right|>\varepsilon\right)=0, \quad \forall \varepsilon>0 .
$$

Definição 8. Dizemos que uma sequência de variáveis aleatórias $\left\{X_{n}\right\}_{n \in \mathbb{N}}$ converge em média $\mathrm{p}$, ou converge em $\mathscr{L}^{p}$ para $X$ se

$$
\lim _{n \rightarrow \infty} \mathbb{E}\left(\left|X_{n}-X\right|^{p}\right)=0
$$

Em particular, quando $p=2$, dizemos que $\left\{X_{n}\right\}_{n \in \mathbb{N}}$ converge em média quadrática.

A próxima e última definição de convergência é chamada convergência em distribuição. Esta é a definição mais fraca de convergência.

Definição 9. Dizemos que uma sequência de variáveis aleatórias $\left\{X_{n}\right\}_{n \in \mathbb{N}}$ converge em distribuição para $X$ se

$$
\lim _{n \rightarrow \infty} F_{X_{n}}(x)=F_{X}(x), \text { para todo } x \text { ponto de continuidade de } F
$$

Segue um exemplo de convergência para variáveis aleatórias:

Exemplo 3. Seja $\left\{Y_{n}\right\}_{n \geq 1}$ uma sequência de variáveis aleatórias tal que

$$
\begin{array}{ll}
\Omega=[0,1], & Y_{n}: \Omega \rightarrow \mathbb{R}, \\
Y_{n}(\omega)=\omega, & \mathbb{P}\left(Y_{n} \leq x\right)=x \quad \text { se } x \in[0,1] .
\end{array}
$$

Isto é, $\left\{Y_{n}\right\}_{n \geq 1}$ é uma sequência de variáveis aleatórias uniformes independentes e identicamente distribuídas.

Defina $X_{n}$ como:

$$
X_{n}= \begin{cases}n, & \text { se } Y_{n} \leq \frac{1}{n^{2}} \\ 0, & \text { caso contrário. }\end{cases}
$$

Podemos reescrever esta variável aleatória da seguinte forma:

$$
X_{n}=\left\{\begin{array}{l}
n, \quad \text { com probabilidade } \frac{1}{n^{2}} \\
0, \quad \text { com probabilidade } 1-\frac{1}{n^{2}}
\end{array}\right.
$$


Note que, para todo $\omega>0$, a sequência $\left\{X_{n}(\omega)\right\}_{n \geq 1}$ converge para zero. Porém, para $\omega=0$, a sequência diverge.

Podemos dizer então que $\left\{X_{n}\right\}_{n \geq 1}$ não converge certamente, mas converge quase certamente, pois $\mathbb{P}(\{\omega \in[0,1]: \omega>0\})=\int_{0}^{1} 1 \mathrm{~d} x=1$.

Faz-se necessário verificar que $\left\{X_{n}\right\}_{n \geq 1}$ converge em probabilidade. Tome $\varepsilon>0$,

$$
\begin{aligned}
\lim _{n \rightarrow \infty} \mathbb{P}\left(\left|X_{n}-0\right|>\varepsilon\right) & =\lim _{n \rightarrow \infty} \mathbb{P}\left(X_{n}=n\right) \\
& =\lim _{n \rightarrow \infty} \frac{1}{n^{2}} \\
& =0 .
\end{aligned}
$$

Portanto, $\left\{X_{n}\right\}_{n \geq 1}$ converge em probabilidade.

Iremos verificar se $X_{n}$ converge em $\mathscr{L}^{1}$ e em média quadrática. Note que

$$
\begin{aligned}
\lim _{n \rightarrow \infty} \mathbb{E}\left(\left|X_{n}-0\right|\right) & =\lim _{n \rightarrow \infty} n \frac{1}{n^{2}} \\
& =\lim _{n \rightarrow \infty} \frac{1}{n} \\
& =0 .
\end{aligned}
$$

Dessa forma,

$$
\begin{aligned}
\lim _{n \rightarrow \infty} \mathbb{E}\left(\left|X_{n}-0\right|^{2}\right) & =\lim _{n \rightarrow \infty} n^{2} \frac{1}{n^{2}} \\
& =1 .
\end{aligned}
$$

Claramente, $X_{n}$ converge em $\mathscr{L}^{1}$ mas não converge em média quadrática.

Outro resultado muito importante quando falamos em convergência é o Lema de Kronecker, que segue:

Lema 1. Seja $\left\{x_{m}\right\}_{m \geq 1}$ uma sequência real finita com $\sum_{m=1}^{\infty} x_{m}=c$, e $\left\{b_{m}\right\}_{m \geq 1}$ uma sequência não decrescente de modo que $\lim _{m \rightarrow \infty} b_{m}=\infty$, então:

$$
\lim _{m \rightarrow \infty} \frac{1}{b_{m}} \sum_{j=1}^{m} b_{j} x_{j}=0 .
$$

Uma vez revisadas tais definições e resultados, podemos falar com maior propriedade sobre a Lei Forte dos Grandes Números e o Teorema Central do Limite. Ross (2010) apresenta de maneira clara os respectivos teoremas (Teorema 2.1 e Teorema 2.2 no livro texto referência). Enunciamos a seguir a Lei Forte dos Grandes Números:

Teorema 1. Seja $\left\{x_{n}\right\}_{n \geq 1}$ uma sequência de variáveis aleatórias independentes e identicamente distribuídas, e seja $\mathbb{E}(X)=\mu$. Então, com probabilidade 1:

$$
\frac{\sum_{i=1}^{n} X_{i}}{n} \rightarrow \mu \text { quando } n \rightarrow \infty
$$


Segue um exemplo da Lei Forte dos Grandes Números:

Exemplo 4. Suponha que, $\left\{x_{n}\right\}_{n \geq 1}$, uma sequência de lançamentos de Bernoulli independentes e identicamente distribuídos ocorra, onde a probabilidade de sucesso de cada lançamento seja $p$, isto é, $\mathbb{P}\left(\left\{X_{i}=1\right\}\right)=p$.

Temos pela Lei Forte dos Grandes Números que, com probabilidade 1:

$$
\frac{\sum_{i=1}^{n} X_{i}}{n} \rightarrow \mathbb{E}(X)=p
$$

Assim como a Lei Forte dos Grandes Números, o Teorema Central do Limite se destaca como um dos mais importantes teoremas da probabilidade.

Teorema 2. Seja $\left\{x_{n}\right\}_{n \geq 1}$ uma sequência de variáveis aleatórias independentes e identicamente distribuídas, com média $\mu$ e variância $\sigma^{2}$. Então, a distribuição de

$$
\frac{\sum_{i=1}^{n} X_{i}-n \mu}{\sigma \sqrt{n}}
$$

tende a uma Normal Padrão quando $n \rightarrow \infty$.

Dessa forma:

$$
\mathbb{P}\left(\frac{\sum_{i=1}^{n} X_{i}-n \mu}{\sigma \sqrt{n}}<\alpha\right) \stackrel{n \rightarrow \infty}{\rightarrow} \frac{1}{\sqrt{2 \pi}} \int_{-\infty}^{\alpha} e^{\frac{-x^{2}}{2}}
$$

Existem implicações entre os tipos de convergência apresentados acima. Karr (1993) apresenta diversas proposições quanto às relações entre os tipos de convergência. Tais proposições são resumidas na figura a seguir.

Figura 1 - Implicações entre tipos de convergência.

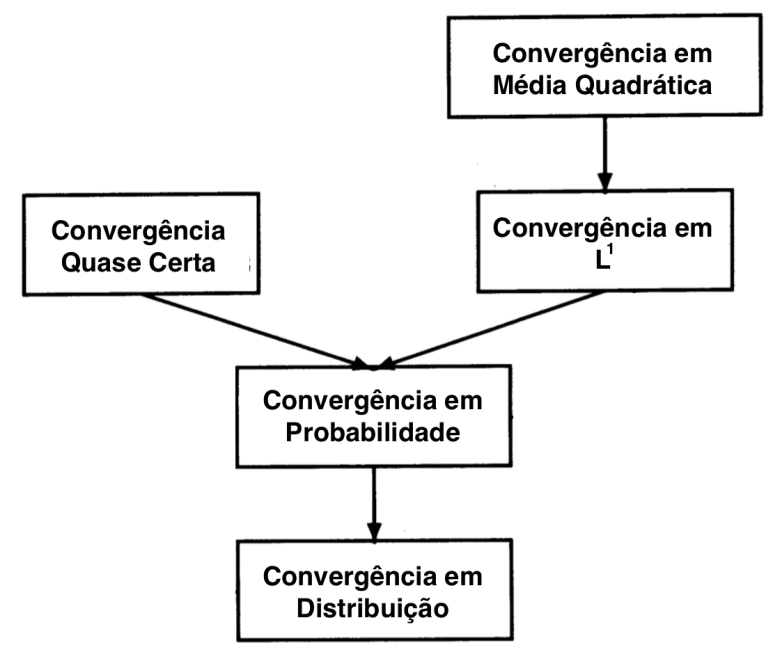




\subsection{Martingales}

O estudo sobre Martingales é de fundamental importância para diversas áreas, como estatística, teoria da probabilidade e matemática financeira.

Nesta seção, serão apresentadas definições, teoremas e corolários referentes à teoria dos martingales com o intuito de aproximar o leitor do tema e mostrar sua aplicabilidade e importância. Para isso, os livros Martingale Limit Theory and Its Application de Hall e Heyde (1980) e Probability with Martingales de Williams (1991) serão utilizados como referência.

Mas o que é de fato um martingale? Podemos ver em Williams (1991) uma definição de Martingale. Para tal leitura, necessitamos antes nos familiarizar com dois termos: filtração e processo adaptado.

Definição 10. Seja $(\Omega, \mathscr{F}, \mathbb{P})$ o espaço de probabilidade.

$\left\{\mathscr{F}_{n}: n \geq 0\right\}$ é uma filtração, isto é, uma família de sub-sigma álgebras de $\mathscr{F}$ :

$$
\mathscr{F}_{0} \subseteq \mathscr{F}_{1} \subseteq \ldots \subseteq \mathscr{F}
$$

Definimos

$$
\mathscr{F}_{\infty}:=\sigma\left(\bigcup_{n} \mathscr{F}_{n}\right) \subseteq \mathscr{F}
$$

Em poucas palavras, podemos dizer que a informação sobre $\omega \in \Omega$ consiste precisamente no valor de $Z(\omega)$ para toda função $Z$ mensurável em $\mathscr{F}_{n}$.

Definição 11. O processo $\left\{x_{n}\right\}_{n \geq 0}$ é chamado de processo adaptado (à filtração $\left\{\mathscr{F}_{n}\right\}$ ) se para cada $n, X_{n}$ for mensurável em $\mathscr{F}_{n}$.

Intuitivamente dizemos que, se $X$ é adaptado, então o valor de $X_{n}(\omega)$ é conhecido no instante $n$.

Com isso podemos seguir com a definição de martingale apresentada por Williams (1991):

Definição 12. O processo $X$ é chamado de martingale (em relação à $\left.\left(\left\{\mathscr{F}_{n}\right\}, \mathbb{P}\right)\right)$ se:

$$
\begin{aligned}
& X \text { é adaptado, } \\
& \mathbb{E}\left(\left|X_{n}\right|\right)<\infty, \forall n, \\
& \mathbb{E}\left(X_{n} \mid \mathscr{F}_{n-1}\right)=X_{n-1}, \text { q.c. }(n \geq 1) .
\end{aligned}
$$

Em palavras, um martingale é uma sequência de variáveis aleatórias (um processo estocástico) para a qual, em um tempo particular na sequência realizada, seu valor esperado em uma próxima realização condicionado aos valores observados é igual ao valor observado no presente. 
Algumas das principais motivações quanto ao estudo de martingales são apostas, jogos de azar e mercado financeiro. Os exemplos a seguir mostram casos onde, claramente, um martingale consegue modelar um jogo de azar.

Exemplo 5. Defina um jogo onde uma moeda justa é lançada a cada instante $n$, isto é, $p=$ probabilidade de cara $=\frac{1}{2}=$ probabilidade de coroa. Se der cara, o apostador recebe $R \$ 1$, caso contrário perde $R \$ 1$. Note que os lançamentos são independentes.

Seja $T_{n}$ o montante de dinheiro que um apostador tem no instante $n$ e $X_{n}$ o resultado da aposta no tempo $n$, sendo $X_{n}=1$ para cara e $X_{n}=-1$ para coroa. Além disso, defina como $\mathscr{F}_{n}$ o histórico de jogadas até o instante $n$.

Note que $T_{n}=\sum_{i=1}^{n} X_{i}$ é mensurável em relação à $\mathscr{F}_{n}$, e,

$$
\mathbb{E}\left(\left|T_{n}\right|\right) \leq n<\infty
$$

Ainda,

$$
\begin{aligned}
\mathbb{E}\left(T_{n+1} \mid \mathscr{F}_{n}\right) & =\mathbb{E}\left(X_{n+1} \mid \mathscr{F}_{n}\right)+T_{n} \\
& =\mathbb{E}\left(X_{n+1}\right)+T_{n} \\
& =1 \times \frac{1}{2}-1 \times \frac{1}{2}+T_{n} \\
& =T_{n} .
\end{aligned}
$$

Portanto, temos que $T_{n}$ é um martingale.

O dinheiro de um apostador é um martingale se todos os jogos de aposta com que ele se envolver forem honestos, pois, para $p \neq \frac{1}{2}$,

$$
\begin{aligned}
\mathbb{E}\left(T_{n+1} \mid \mathscr{F}_{n}\right) & =\mathbb{E}\left(X_{n+1} \mid \mathscr{F}_{n}\right)+T_{n} \\
& =\mathbb{E}\left(X_{n+1}\right)+T_{n} \\
& =1 \times p-1 \times(1-p)+T_{n} \\
& =(2 p-1)+T_{n} \neq T_{n},
\end{aligned}
$$

e portanto $T_{n}$ não é um martingale no caso em que $p \neq \frac{1}{2}$.

Uma vez definido um martingale e exemplificando suas aplicações, podemos seguir em frente para mais uma definição muito utilizada nos estudos com martingales, a Sequência de diferença de martingales, e então seguiremos enunciando três fortes teoremas de convergência para martingales.

Definição 13. Seja $\left\{S_{n}, \mathscr{F}_{n}, n \geq 1\right\}$ um martingale de média zero, quadrado integrável. Seja $X_{n}=S_{n}-S_{n-1}, n \geq 2$ e $X_{1}=S_{1}$, dizemos então que $X_{n}$ é uma diferença de martingales. 
A Definição 13 encontra-se na Seção 3.1 de Hall e Heyde (1980) e seu uso é recorrente na teoria de martingales.

Abordados os conceitos necessários para se enunciar os teoremas de convergência para martingales, iremos apresentá-los ao leitor. Primeiramente, enunciamos o primeiro Teorema de convergência de martingales apresentado por Hall e Heyde (1980) na Seção 1.3:

Teorema 3. Seja, $\left\{Z_{n}, \mathscr{F}_{n}, n \geq 1\right\}$ um submartingale limitado em $L^{1}$. Então existe uma variável $Z$ tal que $\lim _{n \rightarrow \infty} Z_{n}=Z$ q.c. e $\mathbb{E}(|Z|)<\liminf _{n \rightarrow \infty} \mathbb{E}\left(\left|Z_{n}\right|\right)<\infty$. Se o submartingale for uniformemente integrável, então $Z_{n}$ converge para $Z$ em $L^{1}$, e se $\left\{Z_{n}, \mathscr{F}_{n}\right\}$ for um martingale limitado em $L^{2}$, então $Z_{n}$ converge para $Z$ em $L^{2}$.

Em resumo, basta que um martingale $\left\{Z_{n}, \mathscr{F}_{n}\right\}$ seja limitado em $L^{2}$ para que exista $Z$ tal que $Z_{n}$ converge para $Z$ em $L^{2}$.

Hall e Heyde (1980) também apresentam o Teorema de convergência quase certa para martingales (encontra-se no texto referência como Teorema 2.17), apresentado a seguir:

Teorema 4. Seja $\left\{S_{n}=\sum_{i=1}^{n} X_{i}, \mathscr{F}_{n}, n \geq 1\right\}$ um martingale e seja $1 \leq p \leq 2$. Então $S_{n}$ converge quase certamente no conjunto $\left\{\sum_{i=1}^{\infty} \mathbb{E}\left(\left|X_{i}\right|^{p} \mid \mathscr{F}_{i-1}\right)<\infty\right\}$.

Por fim é apresentado o Teorema Central do Limite para martingales e um Corolário. Tal corolário será de fundamental importância para os resultados de James, James e Qi (2008) apresentados posteriormente, em detalhes, no capítulo 4.

Teorema 5. Seja $\left\{S_{n i}, \mathscr{F}_{n i}, 1 \leq i \leq k_{n}, n \geq 1\right\}$ uma sequência de martingales de média zero, com segundo momento finito e com diferença $X_{n i}$, e seja $\eta^{2}$ uma variável aleatória quase certamente finita.

Suponha que

$$
\begin{gathered}
\max _{i}\left|X_{n i}\right| \stackrel{p}{\rightarrow} 0, \\
\sum_{i} X_{n i}^{2} \stackrel{p}{\rightarrow} \eta^{2}, \\
\mathbb{E}\left(\max _{i} X_{n i}^{2}\right) \quad \text { é limitado em } n
\end{gathered}
$$

e as $\sigma$-álgebra são encaixantes:

$$
\mathscr{F}_{n, i} \subseteq \mathscr{F}_{n+1, i} \quad \text { para } \quad 1 \leq i \leq k_{n}, \quad n \geq 1
$$

Então, $S_{n k_{n}}=\sum_{i} X_{n i} \stackrel{d}{\rightarrow} Z$ (em distribuição), onde a variável aleatória $\mathrm{Z}$ tem função característica $\mathbb{E}\left(e^{-\frac{1}{2} \eta^{2} t^{2}}\right)$.

Note que quando $\eta^{2}$ é uma variável aleatória degenerada, $Z \sim N\left(0, \eta^{2}\right)$. 
Em Hall e Heyde (1980), o Teorema 5 mostra $S_{n k_{n}}=\sum_{i} X_{n i} \stackrel{d}{\rightarrow} Z$ estavelmente, porém, a convergência em distribuição é suficiente para os propósitos desta dissertação.

Corolário 1. Se (2.3) e (2.5) são substituídas pela condição condicional de Lindeberg :

$$
\text { para todo } \varepsilon>0, \quad \sum_{i} \mathbb{E}\left[X_{n i}^{2} \mathbb{I}\left(\left|X_{n i}\right|>\varepsilon\right) \mid \mathscr{F}_{n, i-1}\right] \stackrel{p}{\rightarrow} 0,
$$

se (2.4) é substituída por uma condição análoga à variância condicional:

$$
V_{n k_{n}}^{2}=\sum \mathbb{E}\left(X_{n i}^{2} \mid \mathscr{F}_{n, i-1}\right) \stackrel{p}{\rightarrow} \eta^{2}
$$

e se (2.6) se mantém, então a conclusão do Teorema 5 continua verdadeira.

O Teorema 5 e o Corolário 1 são apresentados como Teorema 3.2 e Corolário 3.1 em Hall e Heyde (1980).

Exemplo 6. Suponha $\left\{X_{i}\right\}_{i \geq 1}$ variáveis aleatórias independentes e identicamente distribuídas, com média $\mu<\infty$ e variância $\sigma^{2}<\infty$. Além disso, defina como $\mathscr{F}_{n}$ toda a informação de $\left\{X_{i}\right\}_{i \geq 1}$ até o instante $n$. Iremos demonstrar neste exemplo a Lei Forte dos Grandes Números para variáveis independentes e identicamente distribuídas utilizando os teoremas para martingales apresentados neste capítulo.

Seja $Y_{n}=\sum_{i=1}^{n} X_{i}-\mu n$. Pode-se notar que $Y_{n}$ é mensurável em relação à $\mathscr{F}_{n}$, e,

$$
\mathbb{E}\left(\left|Y_{n}\right|\right)<\infty
$$

Além disso,

$$
\begin{aligned}
\mathbb{E}\left(Y_{n+1} \mid \mathscr{F}_{n}\right) & =\mathbb{E}\left(\sum_{i=1}^{n+1} X_{i}-\mu(n+1) \mid \mathscr{F}_{n}\right) \\
& =\mathbb{E}\left(X_{n+1}+\sum_{i=1}^{n} X_{i}-\mu(n+1) \mid \mathscr{F}_{n}\right) \\
& =\mathbb{E}\left(X_{n+1} \mid \mathscr{F}_{n}\right)+\sum_{i=1}^{n} X_{i}-\mu(n+1) \\
& =\mathbb{E}\left(X_{n+1}\right)+\sum_{i=1}^{n} X_{i}-\mu(n+1) \\
& =\mu+\sum_{i=1}^{n} X_{i}-\mu(n+1) \\
& =\sum_{i=1}^{n} X_{i}-\mu n=Y_{n} .
\end{aligned}
$$

Assim, $Y_{n}$ é um martingale. Seja $T_{n}=Y_{n}-Y_{n-1}$ diferença de Martingales. Note que $T_{n}$ tem média zero e $\mathbb{E}\left(T_{n+1}^{2} \mid \mathscr{F}_{n}\right)=\sigma^{2}$ : 


$$
\begin{aligned}
\mathbb{E}\left(T_{n+1}\right) & =\mathbb{E}\left(Y_{n+1}-Y_{n}\right) \\
& =\mathbb{E}\left(X_{n+1}-\mu\right) \\
& =\mathbb{E}\left(X_{n+1}\right)-\mu \\
& =\mu-\mu=0
\end{aligned}
$$

e,

$$
\begin{aligned}
\mathbb{E}\left(T_{n+1}^{2} \mid \mathscr{F}_{n}\right) & =\mathbb{E}\left(X_{n+1}^{2}-2 \mu X_{n+1}+\mu^{2} \mid \mathscr{F}_{n}\right) \\
& =\mathbb{E}\left(X_{n+1}^{2} \mid \mathscr{F}_{n}\right)-2 \mu \mathbb{E}\left(X_{n+1} \mid \mathscr{F}_{n}\right)+\mu^{2} \\
& =\mathbb{E}\left(X_{n+1}^{2}\right)-2 \mu^{2}+\mu^{2} \\
& =\mathbb{E}\left(X_{n+1}^{2}\right)-\mu^{2}=\sigma^{2}<\infty
\end{aligned}
$$

Sendo assim, tome $Z_{n}=\frac{T_{n}}{n}=\frac{X_{n}-\mu}{n}$. Temos que $\mathbb{E}\left(Z_{n+1}^{2} \mid \mathscr{F}_{n}\right)=\frac{\sigma^{2}}{(n)^{2}}$ e, portanto,

$$
\sum_{i=1}^{\infty} \mathbb{E}\left(Z_{n+1}^{2} \mid \mathscr{F}_{n}\right)=\sigma^{2} \sum_{i=1}^{\infty} \frac{1}{i^{2}}<\infty
$$

Pelo Teorema $4, \sum_{i=1}^{\infty} Z_{i}$ converge quase certamente.

Podemos então, por meio dos resultados obtidos e do lema de Kronecker, obter a Lei Forte dos Grandes Números:

$$
\begin{aligned}
\lim _{n \rightarrow \infty}\left(\frac{\sum_{i=1}^{n} X_{i}}{n}-\mu\right) & =\lim _{n \rightarrow \infty}\left(\frac{\sum_{i=1}^{n} X_{i}-\mu n}{n}\right) \\
& =\lim _{n \rightarrow \infty} \frac{Y_{n}}{n} \\
& =\lim _{n \rightarrow \infty} \frac{\sum_{i=1}^{n} Y_{i}-Y_{i-1}}{n} \\
& =\lim _{n \rightarrow \infty} \frac{\sum_{i=1}^{n} T_{i}}{n} \\
& =\lim _{n \rightarrow \infty} \frac{\sum_{i=1}^{n} \frac{i T_{i}}{i}}{n} \\
& =\lim _{n \rightarrow \infty} \frac{\sum_{i=1}^{n} i Z_{i}}{n}=0
\end{aligned}
$$

Isto é, quando $n \rightarrow \infty, \frac{\sum_{i=1}^{n} X_{i}}{n} \rightarrow \mu$ quase certamente. 



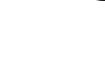

\section{MODELOS PRECURSORES}

Por mais que o modelo apresentado em 2008 por James, James e Qi tenha trazido grandes avanços ao estudo do processo de Bernoulli correlacionado, este se iniciou muito tempo atrás, em 1993, quando Drezner e Farnum (1993) o introduziram.

Tais autores apresentaram um processo de Bernoulli em que a taxa de sucessos no passado consiste em uma proporção da probabilidade de sucesso no presente. Posteriormente, Heyde (2004), apresentou resultados quanto ao comportamento assintótico deste modelo.

A compreensão destes e de outros artigos relacionados são de fundamental importância para um bom entendimento desta dissertação e, consequentemente, do artigo Limit theorems for correlated Bernoulli random variables por James, James e Qi (2008). Portanto, neste capítulo serão introduzidos os resultados obtidos por Drezner e Farnum (1993) e por Heyde (2004) e então serão indicados alguns artigos relacionados para leitura.

\subsection{O primeiro modelo proposto}

Em Drezner e Farnum (1993) e Heyde (2004) considera-se um processo generalizado de Bernoulli $\left\{X_{j}, j \geq 1\right\}$ em que a probabilidade de sucesso a cada lançamento depende da proporção de sucessos nos lançamentos anteriores. Isto é:

$$
\mathbb{P}\left(X_{1}=1\right)=p, \quad \mathbb{P}\left(X_{1}=0\right)=1-p
$$

seja $S_{n}=\sum_{j=1}^{n} X_{j}, n \geq 1$, e $\mathscr{F}_{n}$ a sigma-álgebra gerada por $\left\{X_{n}\right\}_{n \geq 1}$,

$$
\mathbb{P}\left(X_{n+1} \mid \mathscr{F}_{n}\right)=(1-\theta) p+\theta n^{-1} S_{n},
$$

em que $\theta, 0 \leq \theta<1$, é o parâmetro de dependência.

Note que o modelo garante que, se $n^{-1} S_{n}>p(<p)$, então, $\mathbb{P}\left(X_{n+1}=1 \mid \mathscr{F}_{n}\right)>p(<p)$. 


\subsubsection{Resultados de Drezner \& Farnum}

A Binomial generalizada $S_{n}$, estudada por Drezner e Farnum (1993), oferece a possibilidade de se trabalhar com super dispersão quando comparada à binomial clássica. Drezner e Farnum (1993) mostraram que, assim como no caso do processo de Bernoulli simples $(\theta=0)$, $\mathbb{E}\left(X_{j}\right)=p, \mathbb{E}\left(S_{n}\right)=n p$, e enquanto $\operatorname{Var}\left(X_{j}\right)=p(1-p)$,

$$
\mathbb{E}\left[\left(S_{n}-n p\right)^{2}\right]= \begin{cases}p(1-p) n \frac{1-(n \beta(n, 2 \theta))^{-1}}{1-2 \theta}, & \theta \neq \frac{1}{2} \\ p(1-p) n \sum_{j=1}^{n} \frac{1}{j}, & \theta=\frac{1}{2}\end{cases}
$$

onde $\beta$ representa a função Beta dada por: $\beta(n, \phi)=\frac{\Gamma(n) \Gamma(\phi)}{\Gamma(n+\phi)}$.

Dessa forma, utilizando a fórmula de Stirling:

$$
n ! \sim n^{n} e^{-n} \sqrt{2 \pi n} \text {, quando } n \rightarrow \infty
$$

onde, $f(n) \sim g(n)$ significa que $\lim _{n \rightarrow \infty} \frac{f(n)}{g(n)}=1$,

$$
\beta(n, \phi) \sim \Gamma(\phi) n^{-\phi} \text { quando } n \rightarrow \infty \text { para } \phi>0 \text {. }
$$

Logo,

$$
\mathbb{E}\left[\left(S_{n}-n p\right)^{2}\right] \sim \begin{cases}\frac{p(1-p) n}{1-2 \theta}, & \theta<\frac{1}{2}, \\ p(1-p) n \log (n), & \theta=\frac{1}{2} \\ \frac{p(1-p) n^{2 \theta}}{(2 \theta-1) \Gamma(2 \theta)}, & \theta>\frac{1}{2}\end{cases}
$$

e o uso da desigualdade de Chebyshev traz

$$
n^{-1} S_{n} \stackrel{p}{\rightarrow} p \text {, quando } n \rightarrow \infty \text {. }
$$

O comportamento da variância quando $\theta$ difere entre os casos $\theta<\frac{1}{2}, \theta=\frac{1}{2}, \theta>\frac{1}{2}$, o que sugere propriedades interessantes em torno do valor crítico $\frac{1}{2}$.

Exemplo 7. A seguir um exemplo de aplicação fornecido por Drezner e Farnum (1993) para o processo generalizado de Bernoulli. Neste exemplo os parâmetros de dependência não variam, i.e., $\theta_{j}=\theta$ para todo $j$.

Nesta aplicação foram estudados os resultados de 30 temporadas da liga principal de beisebol dos Estados Unidos (de 1961 a 1991, exceto 1981). Cada time jogou 162 vezes por temporada, e no total temos 706 observações sobre o número de vitórias de um determinado time. 
Como o mesmo time joga durante a temporada, é razoável admitir que a uma variável indicadora sobre o time ganhar a $j$-ésima partida está associada ao desempenho do time até o momento da partida de número $j$.

Neste exemplo será comparado o modelo cujos jogos são considerados independentes, ou seja, têm número de vitórias com distribuição binomial, e também o modelo para quando os jogos são considerados dependentes com base no modelo proposto por Drezner e Farnum (1993). Neste caso, a soma de vitórias de um time tem distribuição Binomial generalizada, que denotaremos por "GBD"(Generalized Binomial Distribution).

Quando analisados os dados sobre as 30 temporadas da liga principal de beisebol temos que a média de vitórias é aproximadamente 81 (como esperado), e a variância é dada por 132,595. Note que se o numero total de vitórias de um time em uma temporada tivesse distribuição binomial, seria esperada uma variância de 40,5 $(n p(1-p)$ com $p=1 / 2)$. O problema de variância inflada pode ser resolvido utilizando a abordagem proposta por Drezner e Farnum (1993), como pode ser visto na Figura 2, obtida no artigo em questão (em tal figura os autores utilizaram uma amostra de 20 observações para efeito gráfico).

Figura 2 - GBD aplicado no beisebol.

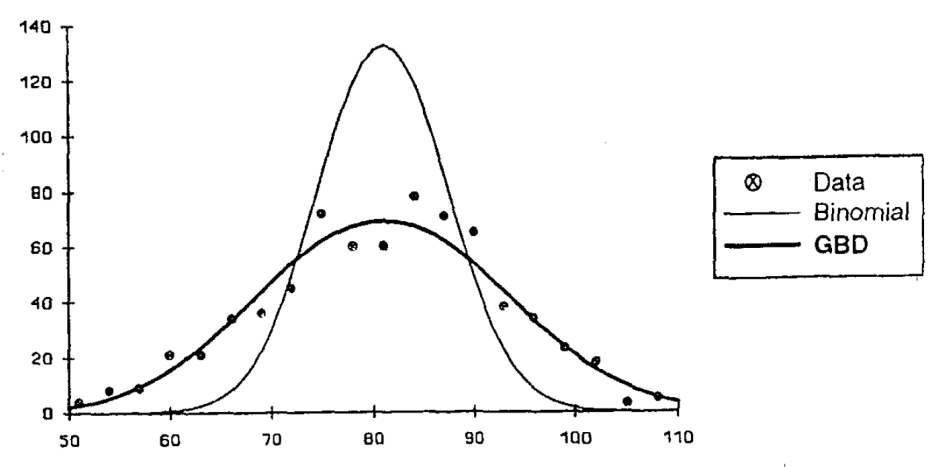

Fonte: Drezner e Farnum (1993)

O modelo Binomial generalizado aparenta ser mais consistente para se tratar usando este banco de dados quando comparado com a abordagem tradicional (considerando os jogos independentes entre si).

\subsubsection{Resultados de Heyde}

Heyde (2004) analisa a taxa de convergência de $n^{-1} S_{n}$ para $p$. Observa-se que o comportamento depende, de maneira crucial, no parâmetro $\theta$, e difere quando $\theta<\frac{1}{2}, \theta=\frac{1}{2}, \theta>\frac{1}{2}$. A normalidade assintótica apenas vale se $\theta \leq \frac{1}{2}$ e a convergência em distribuição é substituída por convergência quase certa se $\theta>\frac{1}{2}$. Os resultados estão resumidos no Teorema (6).

Teorema 6. (i) Se $\theta<\frac{1}{2}$, então $n^{-\frac{1}{2}}\left(S_{n}-n p\right) \stackrel{d}{\rightarrow} N\left(0, \frac{(1-p)}{1-2 \theta}\right)$ quando $n \rightarrow \infty$.

(ii) Se $\theta=\frac{1}{2}$, então $(n \log n)^{-} \frac{1}{2}\left(S_{n}-n p\right) \stackrel{d}{\rightarrow} N(0, p(1-p))$ quando $n \rightarrow \infty$. 
(iii) Se $\theta>\frac{1}{2}$, então $n^{-\theta}\left(S_{n}-n p\right) \stackrel{q . c .}{\rightarrow} W$ quando $n \rightarrow \infty$, em que $W$ é uma variável aleatória tal que:

$$
\mathbb{E}(W)=0, \quad \mathbb{E}\left(W^{2}\right)=\frac{p(1-p)}{(2 \theta-1) \Gamma(2 \theta)}, \quad \text { e } \quad \mathbb{E}\left(W^{3}\right) \neq 0 \quad \text { se } \quad p \neq \frac{1}{2}
$$

Além disso, também foi estabelecida uma dicotomia entre dependência de longo e curto alcance para o processo $\left\{X_{j}\right\}$. Este processo é não estacionário, e a definição padrão de dependência de curto e longo alcance baseada na função de autocovariância do processo não se aplica. Entretanto, a definição mais flexível de Heyde e Yang (1997), baseado na variância amostral de Allen (SAV), que é equivalente à definição clássica, pode ser utilizada. Sob esta definição dizemos que um processo $\left\{Z_{j}\right\}$ de media zero é de curto ou longo alcance se

$$
\frac{\left(\sum_{j=1}^{n} Z_{j}\right)^{2}}{\sum_{j=1}^{n} Z_{j}^{2}} \text { converge ou diverge conforme } n \rightarrow \infty .
$$

Teorema 7. O processo $\left\{X_{j}, j \geq 1\right\}$ tem dependência de curto ou longo alcance (no sentido de SAV) de acordo $\operatorname{com} \theta<\frac{1}{2}$ ou $\theta \geq \frac{1}{2}$. 
Enquanto Drezner e Farnum (1993) apresentaram um modelo no qual o parâmetro de dependência $\theta$ era de mesmo valor para todo instante de tempo $n$, James, James e Qi (2008), apresentaram uma generalização onde o parâmetro de dependência $\theta_{n}$ pode assumir valores distintos tais que $0 \leq \theta_{n} \leq 1$ para todo $n$. Em seu trabalho, os autores demonstram três teoremas e três corolários sobre tal generalização, sendo os teoremas de maior significância a Lei Forte dos Grandes Números e o Teorema Central do Limite para o modelo em questão.

No decorrer deste capítulo apresentaremos o modelo proposto por James, James e Qi (2008) e algumas notações pertinentes para os resultados discorridos. Em seguida, os lemas apresentados por James, James e Qi (2008) que são necessários para realizar as provas do Teorema Central do Limite e da Lei Forte dos Grandes Números serão apresentados e demonstrados. Em sequência, a Lei Forte dos Grandes Números e o Teorema Central do Limite serão enunciados e demonstrados. Por fim, neste capítulo, será enunciada e provada a Lei do Logaritmo Iterado e serão enunciados os demais corolários relacionados a esta lei.

\subsection{Modelo proposto por James, James e Qi (2008)}

Em 'Limit theorems for correlated Bernoulli random variables', James, James e Qi (2008) propuseram um processo de Bernoulli correlacionado de maneira que a probabilidade de sucesso de um lançamento em determinado instante depende da taxa de sucessos dos lançamentos anteriores.

Formalmente, seja $\left\{X_{j}, j \geq 1\right\}$ um processo de Bernoulli de maneira que, para algum $0 \leq p \leq 1$

$$
\mathbb{P}\left(X_{j+1}=1 \mid \mathscr{F}_{n}\right)=\left(1-\theta_{j}\right) p+\theta_{j} \frac{S_{j}}{j}
$$

em que $0 \leq \theta_{j} \leq 1$ são os parâmetros de dependência, $S_{n}=\sum_{j=1}^{n} X_{j}$ e $\mathscr{F}_{n}$ a sigma-álgebra gerada até o instante $n$. 
Como visto no Capítulo 3, Drezner e Farnum (1993) introduziram a distribuição de $S_{n}$ como Distribuição Binomial Generalizada. Além disso, Heyde (2004) demonstrou propriedades do comportamento assintótico de um caso particular deste modelo, onde $\theta_{j}=\theta$ para todo $j \geq 1$, $\theta \in[0,1)$.

Uma vez que os resultados apresentados por James, James e Qi (2008) se referem ao comportamento assintótico do Processo de Bernoulli Correlacionado, algumas definições se fazem necessárias para uma boa leitura do texto.

Definimos então as seguintes funções do parâmetro $\theta_{n}$ :

$$
a_{1}=1, \quad a_{n}=\prod_{i=1}^{n-1}\left(1-i^{-1} \theta_{i}\right) \text { para } n \geq 2
$$

$\mathrm{e}$

$$
A_{n}^{2}=\sum_{j=1}^{n} a_{j}^{-2} \text { para } n \geq 1
$$

Para além dessas, as seguintes funções serão abordadas no decorrer do capítulo:

$$
S_{n}=\sum_{i=1}^{n} X_{i}
$$

e

$$
T_{n}=\frac{S_{n}-n p}{a_{n}}
$$

Por fim, definimos $Y_{1}=T_{1}$ e $Y_{n}=T_{n}-T_{n-1}$.

\subsection{Lemas}

Em seu artigo, James, James e Qi (2008) apresentam alguns lemas que são utilizados para as demonstrações dos teoremas e corolários. Nesta dissertação são apresentados tais lemas assim como suas demonstrações.

Iremos apresentar os lemas de 2 a 6 abaixo de maneira que na prova da Lei Forte dos Grandes Números serão utilizados os lemas 3 e 4, na prova do Teorema Central do Limite serão utilizados os lemas 4 e 5, na prova da Lei do Logaritmo Iterado será utilizado o lema 6 e nas provas dos corolários será utilizado o lema 2.

Lema 2. Para qualquer $\theta>0, a_{n}(\theta) \sim \frac{n^{\theta}}{\theta \Gamma(\theta)}$ quando $n \rightarrow \infty$, onde $a_{n}(\theta)$ é $a_{n}$ quando $\theta_{n}=\theta$ para todo $n \geq 1$.

Demonstração. Seja $\theta>0$,

$$
a_{n}(\theta)=\prod_{j=1}^{n-1}\left(1+j^{-1} \theta\right)=\prod_{j=1}^{n-1} \frac{j+\theta}{j}=\frac{\prod_{j=1}^{n-1}(j+\theta)}{\Gamma(n)} .
$$


Note que

$$
\begin{aligned}
& \prod_{j=1}^{n-1}(j+\theta)=(n-1+\theta)(n-2+\theta) \ldots(1+\theta) \\
= & (n-1+\theta)(n-2+\theta) \ldots(1+\theta) \frac{\theta}{\theta} \frac{\Gamma(\theta)}{\Gamma(\theta)}=\frac{\Gamma(\theta+n)}{\theta \Gamma(\theta)} ;
\end{aligned}
$$

$\log$,

$$
a_{n}(\theta)=\frac{\Gamma(\theta+n)}{\theta \Gamma(\theta) \Gamma(n)}=\frac{1}{\theta \beta(n, \theta)},
$$

onde $\beta(n, \theta)=\frac{\Gamma(n+\theta)}{\Gamma(n) \Gamma(\theta)}$.

Através da fórmula de Stirling $\left(n ! \sim n^{n} e^{-n} \sqrt{2 \pi n}\right.$, quando $\left.n \rightarrow \infty\right)$ podemos observar que $\beta(n, \theta) \sim \Gamma(\theta) n^{-\theta}$. Portanto,

$$
a_{n}(\theta) \sim \frac{n^{\theta}}{\theta \Gamma(\theta)}
$$

Com isso, seguimos para o próximo lema que será utilizado na Lei Forte dos Grandes Números.

Lema 3. (i) $\frac{n}{a_{n}}$ é não decrescente em $n$,

(ii) Se $\lim _{n \rightarrow \infty} A_{n}=\infty$

Então, $\lim _{n \rightarrow \infty} \frac{a_{n}}{n}=0$

Demonstração. Para tal prova iremos utilizar a seguinte notação:

$$
a_{n}(\theta)=\prod_{j=1}^{n-1}\left(1+\frac{\theta}{j}\right)
$$

Primeiramente, note que

$$
a_{n}(1)=\prod_{j=1}^{n-1}\left(1+\frac{1}{j}\right)=\prod_{j=1}^{n-1}\left(\frac{j+1}{j}\right)=\frac{2}{1} \frac{3}{2} \frac{4}{3} \times \ldots \times \frac{n-1}{n-2} \frac{n}{n-1}=n,
$$

para todo $n \geq 1$. Assim temos que

$$
\frac{n}{a_{n}}=\frac{a_{n}(1)}{a_{n}}=\prod_{j=1}^{n-1} \frac{1+j^{-1}}{1+j^{-1} \theta_{j}},
$$

que é não-decrescente já que todos os termos do produto em (4.1) são ao menos 1. Com isso concluímos a parte (i) do lema.

Para prova (ii), suponha que $\lim _{n \rightarrow \infty} A_{n}=\infty$. 
Como $\frac{n}{a_{n}}$ é positiva e não-decrescente, então $\frac{a_{n}}{n}$ é positiva e não-crescente, e assim, por ser limitada entre 0 e $a_{1}$, seu limite existe. Chame este limite de $v$.

Suponha que $v>0$. Com isso temos:

$$
\frac{a_{n}}{n}>v \Longleftrightarrow a_{n}>n v \Longleftrightarrow \frac{1}{a_{n}}<\frac{1}{n v} \Longleftrightarrow \frac{1}{a_{n}^{2}}<\frac{1}{n^{2} v^{2}} \text {, para todo } n \geq 1 ;
$$

e portanto,

$$
\lim _{n \rightarrow \infty} A_{n}^{2}=\sum_{n=1}^{\infty} \frac{1}{a_{n}^{2}}<\frac{1}{v^{2}} \sum_{n=1}^{\infty} \frac{1}{n^{2}}<\infty,
$$

o que é absurdo pois partimos do pressuposto que $\lim _{n \rightarrow \infty} A_{n}=\infty$.

Sendo assim, $v$ precisa ser zero. Concluímos então a segunda parte do lema.

O próximo lema, que será de grande importância tanto na demonstração da Lei Forte dos Grandes Números quanto para o Teorema Central do Limite, é provado a seguir:

Lema 4. Seja $\left\{Z_{n}, \mathscr{F}_{n}, n \geq 1\right\}$ uma sequência de diferenças de martingales.

Se $\sum_{j=1}^{\infty} \mathbb{E}\left(Z_{j}^{2} \mid \mathscr{F}_{j-1}\right)<\infty$ quase certamente, então $\sum_{j=1}^{n} Z_{j}$ converge quase certamente.

Demonstração. Para provarmos este lema precisamos relembrar o Teorema 4.

Nele dizemos que, se $S_{n}=\sum_{i=1}^{n} X_{i}$ é um martingale, então para $1 \leq p \leq 2, S_{n}$ converge quase certamente no conjunto $\left\{\sum_{i=1}^{\infty} \mathbb{E}\left(\left|X_{i}\right|^{p} \mid \mathscr{F}_{i-1}\right)<\infty\right\}$.

Note que a suposição de que $\sum_{j=1}^{\infty} \mathbb{E}\left(Z_{j}^{2} \mid \mathscr{F}_{j-1}\right)<\infty$ quase certamente, presente no Lema 4, é a condição necessária apresentada no Teorema 4.

Sendo assim, o Lema 4 é um caso particular do Teorema 4 para quando $p=2$. Concluímos, então, a prova do lema.

O Lema 5, utilizado na demonstração do Teorema Central do Limite, é enunciado e provado a seguir.

Lema 5. Seja $\left\{Z_{n}, \mathscr{F}_{n}, n \geq 1\right\}$ uma sequência de diferenças de martingales limitada.

Assuma que exista uma sequência de constantes positivas $\left\{W_{n}\right\}$ de maneira que $W_{n} \rightarrow \infty$ quando $n \rightarrow \infty \mathrm{e}$

$$
W_{n}^{-2} \sum_{i=1}^{n} \mathbb{E}\left(Z_{i}^{2} \mid \mathscr{F}_{i-1}\right) \stackrel{p}{\rightarrow} \sigma^{2} .
$$

Então

$$
W_{n}^{-1} \sum_{j=1}^{n} Z_{n} \stackrel{d}{\rightarrow} N\left(0, \sigma^{2}\right) .
$$


Demonstração. Para realizar tal demonstração precisamos relembrar o Corolário 1.

Nele temos que, de maneira simplificada, dada uma sequência de diferenças de martingales $X_{i}$, se a condição condicional de Lindeberg for satisfeita, isto é

$$
\text { para todo } \varepsilon>0, \quad \sum_{i} \mathbb{E}\left[X_{i}^{2} \mathbb{I}\left(\left|X_{i}\right|>\varepsilon\right) \mid \mathscr{F}_{i-1}\right] \stackrel{p}{\rightarrow} 0
$$

e

$$
\sum \mathbb{E}\left(X_{i}^{2} \mid \mathscr{F}_{i-1}\right) \stackrel{p}{\rightarrow} \eta^{2} \text { (sendo } \eta \text {, neste caso, uma v.a. degenerada), }
$$

então

$$
\sum_{i} X_{i} \stackrel{d}{\rightarrow} N\left(0, \eta^{2}\right)
$$

Tendo em vista tal corolário, definimos $Z_{n i}=\frac{Z_{i}}{W_{n}}$ para $1 \leq i \leq n$. Note que a suposição assumida no Lema 5 equivale à equação (4.3) e, portanto, basta provar que a condição condicional de Lindeberg vale, isto é

$$
\sum_{i=1}^{n} \mathbb{E}\left(Z_{n i} \mathbb{I}\left(Z_{n i}>\varepsilon\right) \mid \mathscr{F}_{i-1}\right) \stackrel{p}{\rightarrow} 0 \text { para todo } \varepsilon>0
$$

o que é trivial uma vez que, para qualquer $\varepsilon>0$, todo conjunto $\left\{Z_{n i}>\varepsilon\right\}$ é vazio para $n$ grande.

Por fim, o Lema 6, que tem fundamental importância para a prova do Teorema 10, presente na seção 4.6 do capítulo 4, é enunciado.

Lema 6. Seja $\left\{Z_{n}, \mathscr{F}_{n}, n \geq 1\right\}$ uma sequência de diferenças de martingales limitadas. Assuma que exista uma sequência de constantes positivas $W_{n}$ tal que:

$$
\begin{aligned}
& \frac{W_{n}}{W_{n+1}} \rightarrow 1, \\
& W_{n} \rightarrow \infty,
\end{aligned}
$$

$\mathrm{e}$

$$
\frac{1}{W_{n}^{2}} \sum_{j=1}^{n} \mathbb{E}\left(Z_{j} \mid \mathbb{F}_{j-1}\right) \stackrel{\text { q.c. }}{\rightarrow} 1
$$

Então,

$$
\limsup _{n \rightarrow \infty} \pm \frac{\sum_{j=1}^{n} Z_{j}}{\sqrt{2 W_{n}^{2} \log \log W_{n}^{2}}} \stackrel{\text { q.c. }}{=} 1 .
$$

Para maior entendimento do lema acima é recomendada a leitura dos teoremas 4.7 e 4.8 de Hall e Heyde (1980).

Uma vez enunciados os lemas, apresentamos agora a Lei Forte dos Grandes Números e Teorema Central do Limite e suas respectivas provas. O capítulo será finalizado com o enunciado e prova do Teorema 10 e os demais corolários presentes no artigo. 


\subsection{Lei Forte dos Grandes Números}

No artigo de James, James e Qi (2008) é demonstrada a Lei Forte dos Grandes Números para o modelo e uma condição necessária para a validade da lei.

Nesta seção iremos enunciar tal teorema e provar sua primeira parte. A sua reciprocidade será provada na seção 4.5 .

Teorema 8. Se $\lim _{n \rightarrow \infty} \frac{a_{n}}{n}=0$, então $\frac{S_{n}}{n} \stackrel{q . c .}{=} p$.

Reciprocamente, se $\frac{S_{n}}{n} \stackrel{q . c .}{=} p$, então $\lim _{n \rightarrow \infty} \frac{a_{n}}{n}=0$.

Demonstração. Para mostrar a parte suficiente da Lei Forte dos Grandes Números, isto é, que dada a condição $\lim _{n \rightarrow \infty} \frac{a_{n}}{n}=0$, então $\frac{S_{n}}{n} \stackrel{q . c .}{=} p$, primeiramente devemos mostrar que $\left\{T_{n}, \mathscr{F}_{n}, n \geq 1\right\}$ é uma sequência de martingales.

Lembre que $T_{n}=\frac{S_{n}-n p}{a_{n}}$, e note que

$$
\begin{aligned}
a_{n} & =\prod_{i=1}^{n-1}\left(1+\frac{\theta_{i}}{i}\right)=\prod_{i=1}^{n-1} \frac{i+\theta_{i}}{i} \\
& =\frac{n-1+\theta_{n-1}}{n-1} \prod_{i=1}^{n-2} \frac{i+\theta_{i}}{i}=a_{n-1}\left(1+\frac{\theta_{n}}{n-1}\right)
\end{aligned}
$$

$\log$,

$$
\frac{1}{a_{n}}=\frac{1}{a_{n-1}}\left(\frac{n-1}{n-1+\theta_{n}}\right) \Longleftrightarrow \frac{1}{a_{n}}\left(\frac{n-1+\theta_{n}}{n-1}\right)=\frac{1}{a_{n-1}} .
$$

Então,

$$
\begin{aligned}
\mathbb{E}\left(T_{n} \mid \mathscr{F}_{n-1}\right) & =\mathbb{E}\left(\frac{S_{n}-n p}{a_{n}} \mid \mathscr{F}_{n-1}\right) \\
& =\frac{1}{a_{n}}\left(\mathbb{E}\left(S_{n} \mid \mathscr{F}_{n-1}\right)-n p\right) \\
& =\frac{1}{a_{n}}\left(\mathbb{E}\left(X_{n} \mid \mathscr{F}_{n-1}\right)+S_{n-1}-n p\right) \\
& =\frac{1}{a_{n}}\left(\mathbb{P}\left(X_{n}=1 \mid \mathscr{F}_{n-1}\right)+S_{n-1}-n p\right) \\
& =\frac{1}{a_{n}}\left(\left(1-\theta_{n-1}\right) p+\theta_{n-1} \frac{S_{n-1}}{n-1}+S_{n-1}-n p\right) \\
& =\frac{1}{a_{n}}\left(\left(1-\theta_{n-1}-n\right) p+\frac{n-1+\theta_{n-1}}{n-1} S_{n-1}\right) \\
& =\frac{1}{a_{n}} \frac{n-1+\theta_{n-1}}{n-1}\left(S_{n-1}-(n-1) p\right) \\
& =\frac{S_{n-1}-(n-1) p}{a_{n-1}} \\
& =T_{n-1} .
\end{aligned}
$$


Uma vez mostrado que $T_{n}$ é martingale,

Seja $Y_{n}, n \geq 1$ as diferenças do martingale $\left\{T_{n}\right\}$, para $j>1$ :

$$
\begin{aligned}
Y_{j} & =T_{j}-T_{j-1}=\frac{S_{j}-j p}{a_{j}}-\frac{S_{j-1}-(j-1) p}{a_{j-1}} \\
& =\frac{X_{j}-S_{j-1}-j p}{a_{j}}-\left(1+\frac{\theta_{j-1}}{j-1}\right) \times \frac{S_{j-1}-(j-1) p}{a_{j}} \\
& =\frac{1}{a_{j}}\left(X_{j}+S_{j-1}-j p-S_{j-1}+(j-1) p-\frac{\theta_{j-1} S_{j-1}}{j-1}+\theta_{j-1} p\right) \\
& =\frac{1}{a_{j}}\left(X_{j}-p+\theta_{j-1}\left(p-\frac{S_{j-1}}{j-1}\right)\right) .
\end{aligned}
$$

Note que:

$$
\mathbb{E}\left(Y_{j}^{2} \mid \mathscr{F}_{j-1}\right)=\frac{1}{a_{j}^{2}}\left(p(1-p)+\theta_{j-1}(1-2 p)\left(\frac{S_{j-1}}{j-1}-p\right)-\theta_{j-1}^{2}\left(\frac{S_{j-1}}{j-1}-p\right)^{2}\right)
$$

e como $0 \leq S_{j-1} \leq j-1$, existe uma constante $C$ tal que:

$$
\mathbb{E}\left(Y_{j}^{2} \mid \mathscr{F}_{j-1}\right) \leq \frac{C}{a_{j}^{2}}, \text { para } j>1
$$

Seja $Z_{n}=\frac{a_{n} Y_{n}}{n}, n \geq 1$, então $\left\{Z_{n}, \mathscr{F}_{n}, n \geq 1\right\}$ é uma sequência de diferença dos martingales limitados.

De (4.5):

$$
\begin{aligned}
& \mathbb{E}\left(Z_{j}^{2} \mid \mathscr{F}_{j-1}\right)=\mathbb{E}\left(\frac{a_{j}^{2} Y_{j}^{2}}{j^{2}} \mid \mathscr{F}_{j-1}\right) \\
= & \frac{a_{j}^{2}}{j^{2}} \mathbb{E}\left(Y_{j}^{2} \mid \mathscr{F}_{j-1}\right) \leq C \frac{1}{j^{2}}, \text { para } j>1 .
\end{aligned}
$$

Então,

$$
\sum_{j=2}^{\infty} \mathbb{E}\left(Z_{j}^{2} \mid \mathscr{F}_{j-1}\right) \leq C \sum_{j=2}^{\infty} \frac{1}{j^{2}}<\infty
$$

e portanto, pelo Lema 4,

$$
\sum_{j=1}^{n} \frac{a_{j} Y_{j}}{j}=\sum_{j=1}^{n} Z_{j} \text { converge quase certamente. }
$$

Como $n / a_{n}$ é não decrescente e vai para o infinito, segue pelo Lema 3 e pelo Lema 1 (lema de 
Kronecker):

$$
\begin{aligned}
\lim _{n \rightarrow \infty}\left|\frac{S_{n}}{n}-p\right| & =\lim _{n \rightarrow \infty}\left|\frac{S_{n}-n p}{n}\right| \\
& =\lim _{n \rightarrow \infty}\left|\frac{a_{n} T_{n}}{n}\right| \\
& =\lim _{n \rightarrow \infty}\left|\frac{\sum_{j=1}^{n} Y_{j}}{n / a_{n}}\right| \\
& =\lim _{n \rightarrow \infty}\left|\frac{\sum_{j=1}^{n} \frac{j}{a_{j}} \frac{a_{j}}{j} Y_{j}}{n / a_{n}}\right| \\
& =\lim _{n \rightarrow \infty}\left|\frac{\sum_{j=1}^{n} \frac{j}{a_{j}} Z_{j}}{n / a_{n}}\right| \stackrel{q . c .}{=} 0 .
\end{aligned}
$$

Assim, a parte suficiente do Teorema (8) está provada.

Para demonstrar a sua reciprocidade, isto é, se $\frac{S_{n}}{n} \stackrel{\text { q.c. }}{=} p$ então $\lim _{n \rightarrow \infty} \frac{a_{n}}{n}=0$, necessitamos demonstrar primeiro o Teorema Central do Limite, o que faremos na próxima seção.

\subsection{Teorema Central do Limite}

Dentre os teoremas provados por James, James e Qi (2008) no artigo estudado nesta dissertação, destaca-se o Teorema Central do Limite para o Processo de Bernoulli Correlacionado. Para realizar sua prova é necessário utilizar a parte suficiente da Lei Forte dos Grandes Números (Teorema 8). Ademais, o Teorema Central do Limite se faz necessário para realizar a prova da reciprocidade da Lei Forte dos Grandes Números.

Antes de enunciarmos o teorema e realizarmos sua demonstração é conveniente introduzir a notação de $o(g(x))$ (lê-se ó pequeno).

Definição 14. Dizemos que uma função $f(x)$ é $o(g(x))$ se

$$
\lim _{x \rightarrow \infty} \frac{f(x)}{g(x)}=0 \text {. }
$$

Uma vez definido o conceito de $o(g(x))$, seguimos com o enunciado do Teorema Central do Limite para o Processo de Bernoulli Correlacionado, seguido de sua demonstração.

Teorema 9. Se $\lim _{n \rightarrow \infty} A_{n}=\infty$, então

$$
\frac{S_{n}-n p}{A_{n} a_{n}} \stackrel{d}{\rightarrow} N(0, p(1-p))
$$

Já, se $\lim _{n \rightarrow \infty} A_{n}<\infty$, então

$$
\frac{S_{n}-n p}{a_{n}} \stackrel{q \cdot c .}{\rightarrow} V
$$

em que $V$ é uma variável aleatória não degenerada com média diferente de zero. 
Demonstração. Primeiramente, assuma $\lim _{n \rightarrow \infty} A_{n}=\infty$. Pelo lema $3, \lim _{n \rightarrow \infty} \frac{a_{n}}{n}=0$. Sobre estas condições temos de (4.4):

$$
\mathbb{E}\left(Y_{j}^{2} \mid \mathscr{F}_{j-1}\right)=\frac{1}{a_{j}^{2}}\left(p(1-p)+\theta_{j-1}(1-2 p)\left(\frac{S_{j-1}}{j-1}-p\right)-\theta_{j-1}^{2}\left(\frac{S_{j-1}}{j-1}-p\right)^{2}\right)
$$

e, utilizando o teorema 8 (apenas a parte suficiente), sabemos que $\left(\frac{s_{j-1}}{j-1}-p\right)$ é $o(1)$ e assim

$$
\mathbb{E}\left(Y_{j}^{2} \mid \mathscr{F}_{j-1}\right)=\frac{p(1-p)}{a_{j}^{2}}(1+o(1)) \text { quando } j \rightarrow \infty
$$

com probabilidade 1. Portanto, de (4.9) e com a suposição de que $\lim _{n \rightarrow \infty} A_{n}=\infty$,

$$
\begin{aligned}
& \lim _{n \rightarrow \infty} \frac{\sum_{j=1}^{n} \mathbb{E}\left(Y_{j}^{2} \mid \mathscr{F}_{j-1}\right)}{A_{n}^{2}}=\lim _{n \rightarrow \infty} \frac{\sum_{j=1}^{n}\left(\frac{p(1-p)}{a_{j}^{2}}(1+o(1))\right)}{A_{n}^{2}} \\
= & p(1-p)\left(\frac{(1+o(1)) A_{n}^{2}}{A_{n}^{2}}\right) \stackrel{q . c .}{=} p(1-p) .
\end{aligned}
$$

Logo, pelo lema 5,

$$
\frac{S_{n}-n p}{a_{n} A_{n}}=\frac{T_{n}}{A_{n}}=\frac{\sum_{j=1}^{n} Y_{j}}{A_{n}} \stackrel{d}{\rightarrow} N(0, p(1-p))
$$

provando (4.7) (a primeira parte do teorema 9).

Partimos agora para a segunda parte do teorema 9, que diz que se $\lim _{n \rightarrow \infty} A_{n}<\infty$, então

$$
\frac{S_{n}-n p}{a_{n}} \stackrel{q \cdot c .}{\rightarrow} V
$$

Para isso, assuma $\lim _{n \rightarrow \infty} A_{n}<\infty$. De (4.6) temos

$$
\sum_{j=1}^{\infty} \mathbb{E}\left(Y_{j}^{2} \mid \mathscr{F}_{j-1}\right) \leq C \sum_{j=1}^{\infty} \frac{1}{a_{j}^{2}}<\infty, \text { q.c. }
$$

Pelo lema 4,

$$
T_{n}=\frac{S_{n}-n p}{a_{n}}=\sum_{j=1}^{n} Y_{j} \text { converge q.c. para uma variável aleatória } V \text {. }
$$


Note que $\mathbb{E}\left(\sum_{j=1}^{n} Y_{j}\right)=0 \mathrm{e}$

$$
\begin{aligned}
& V\left(\sum_{j=1}^{n} Y_{j}\right)=\mathbb{E}\left(\sum_{j=1}^{n} Y_{j}\right)^{2}-\mathbb{E}^{2}\left(\sum_{j=1}^{n} Y_{j}\right) \\
= & \mathbb{E}\left(\sum_{j=1}^{n} Y_{j}\right)^{2}=\mathbb{E}\left(\sum_{j=1}^{n} \sum_{i=1}^{n} Y_{j} Y_{i}\right) \\
= & \mathbb{E}\left(\sum_{j=1}^{n} Y_{j}^{2}\right)+2 \mathbb{E}\left(\sum_{j>i}^{n} Y_{j} Y_{i}\right) \\
= & \sum_{j=1}^{n} \mathbb{E}\left(Y_{j}^{2}\right)+2 \sum_{j>i}^{n} \mathbb{E}\left(Y_{i} \mathbb{E}\left(Y_{j} \mid \mathscr{F}_{i}\right)\right) \\
= & \sum_{j=1}^{n} \mathbb{E}\left(Y_{j}^{2}\right),
\end{aligned}
$$

como $\mathbb{E}\left(Y_{j} \mid \mathscr{F}_{i}\right)=0$, para $j>i$, então $V$ é uma variável aleatória de média zero e variância positiva igual a $\sum_{j=1}^{n} \mathbb{E}\left(Y_{j}^{2}\right)$. Concluímos a prova do Teorema 9.

\subsection{Reciprocidade da Lei Forte dos Grandes Números}

Uma vez enunciado e provado o Teorema Central do Limite podemos voltar para a reciprocidade da Lei Forte dos Grandes Números, isto é, se $\frac{S_{n}}{n} \stackrel{q . c .}{=} p$, então

$$
\lim _{n \rightarrow \infty} \frac{a_{n}}{n}=0
$$

Para isso, vamos assumir que

$$
\frac{S_{n}}{n} \stackrel{q . c .}{=} p
$$

Suponha que $\lim _{n \rightarrow \infty} a_{n} / n \nrightarrow 0$, então pelo Lema 3,

$$
\begin{aligned}
\left(\lim _{n \rightarrow \infty} A_{n}=\infty\right) & \Longrightarrow\left(\frac{a_{n}}{n} \rightarrow 0\right) \\
\Longleftrightarrow \lim _{n \rightarrow \infty} a_{n} / n \nrightarrow 0 & \Longrightarrow \lim _{n \rightarrow \infty} A_{n}<\infty .
\end{aligned}
$$

Temos então que $\lim _{n \rightarrow \infty} A_{n}<\infty$. Com isso, pelo Teorema $9,\left(\frac{S_{n}}{n}-p\right) \stackrel{q . c .}{\rightarrow} V$, em que $V$ é uma variável aleatória não degenerada, o que é absurdo, pois vai contra a suposição (4.13).

E isto completa a prova da reciprocidade do Teorema 8.

\subsection{Lei do Logaritmo Iterado}

Além dos resultados minuciosamente detalhados nesta dissertação, James, James e Qi (2008) ainda apresentaram um teorema e dois corolários. Tais resultados são apresentados a seguir. 
Teorema 10. Se $\lim _{n \rightarrow \infty} A_{n}=\infty$, então

$$
\limsup _{n \rightarrow \infty} \pm \frac{S_{n}-n p}{a_{n} A_{n} \sqrt{\log \log A_{n}}} \stackrel{q . c .}{=} \sqrt{2 p(1-p)} .
$$

Demonstração. Para provarmos o Teorema 10 utilizaremos o resultado do Lema 6 que, em poucas palavras, diz que dado $\left\{Z_{n}, \mathscr{F}_{n}, n \geq 1\right\}$ uma sequência de diferenças de martingales limitadas e $W_{n}$ uma sequência de constantes positivas, se:

$$
\begin{aligned}
& \frac{W_{n}}{W_{n+1}} \rightarrow 1, \\
& W_{n} \rightarrow \infty,
\end{aligned}
$$

e

$$
\frac{1}{W_{n}^{2}} \sum_{j=1}^{n} \mathbb{E}\left(Z_{j} \mid \mathscr{F}_{j-1}\right) \stackrel{\text { q.c. }}{\rightarrow} 1,
$$

então,

$$
\limsup _{n \rightarrow \infty} \pm \frac{\sum_{j=1}^{n} Z_{j}}{\sqrt{2 W_{n}^{2} \log \log W_{n}^{2}}} \stackrel{\text { q.c. }}{=} 1 .
$$

Com isso, considere $Z_{n}=Y_{n}$ e $W_{n}^{2}=A_{n}^{2} p(1-p)$. Pode-se ver que, uma vez que $0<p(1-p)<1$ e que partimos da suposição de que $A_{n}^{2} \rightarrow \infty, A_{n}^{2} p(1-p)=W_{n} \rightarrow \infty$, então

$$
\begin{aligned}
\lim _{n \rightarrow \infty} \frac{W_{n}}{W_{n+1}} & =\lim _{n \rightarrow \infty} \frac{A_{n}^{2} p(1-p)}{A_{n+1}^{2} p(1-p)} \\
& =\lim _{n \rightarrow \infty} \frac{A_{n}^{2} p(1-p)}{a_{n+1}^{-2} p(1-p)+A_{n}^{2} p(1-p)} \\
& =\lim _{n \rightarrow \infty} \frac{A_{n}^{2} p(1-p)}{A_{n}^{2} p(1-p)} \frac{1}{1+\frac{a_{n+1}^{-2}}{A_{n}^{2}}} \\
& =1 .
\end{aligned}
$$

É ainda necessário mostrar que $\frac{1}{W_{n}^{2}} \sum_{j=1}^{n} \mathbb{E}\left(Z_{j} \mid \mathscr{F}_{j-1}\right) \stackrel{q . c .}{\rightarrow} 1$. Para isso, utilizamos a equação (4.10) que diz que

$$
\lim _{n \rightarrow \infty} \frac{\sum_{j=1}^{n} \mathbb{E}\left(Y_{j}^{2} \mid \mathscr{F}_{j-1}\right)}{A_{n}^{2}} \stackrel{\text { q.c. }}{=} p(1-p) .
$$

Assim, temos que

$$
\begin{aligned}
\lim _{n \rightarrow \infty} \frac{\sum_{j=1}^{n} \mathbb{E}\left(Z_{j}^{2} \mid \mathscr{F}_{j-1}\right)}{W_{n}^{2}} & =\lim _{n \rightarrow \infty} \frac{\sum_{j=1}^{n} \mathbb{E}\left(Y_{j}^{2} \mid \mathscr{F}_{j-1}\right)}{A_{n}^{2} p(1-p)} \\
& \stackrel{\text { q.c. }}{=} \frac{p(1-p)}{p(1-p)}=1
\end{aligned}
$$


e, pelo Lema 6:

$$
\begin{aligned}
\limsup _{n \rightarrow \infty} \pm \frac{\sum_{j=1}^{n} Z_{j}}{\sqrt{2 W_{n}^{2} \log \log W_{n}^{2}}} & =\limsup _{n \rightarrow \infty} \pm \frac{\sum_{j=1}^{n} Y_{j}}{\sqrt{2 A_{n}^{2} p(1-p) \log \log \left(A_{n}^{2} p(1-p)\right)}} \\
& =\limsup _{n \rightarrow \infty} \pm \frac{S_{n}-n p}{a_{n} A_{n} \sqrt{2 p(1-p) \log \log \left(A_{n}^{2} p(1-p)\right)}} \\
& \stackrel{\text { q.c. }}{=} 1 .
\end{aligned}
$$

Para concluir, precisamos mostrar que $\log \log A_{n}^{2} \sim \log \log W_{n}^{2}$, isto é,

$$
\lim _{n \rightarrow \infty} \frac{\log \log A_{n}^{2}}{\log \log W_{n}^{2}}=1 .
$$

Seguindo, considere $\log (p(1-p))=k$ :

$$
\begin{aligned}
& \lim _{n \rightarrow \infty} \frac{\log \log A_{n}^{2}}{\log \log W_{n}^{2}}=\lim _{n \rightarrow \infty} \frac{\log \log A_{n}^{2}}{\log \log \left(A_{n}^{2} p(1-p)\right)} \\
& =\lim _{n \rightarrow \infty} \frac{\log \log A_{n}^{2}}{\log \left(\log \left(A_{n}^{2}\right)+k\right)} \\
& =\lim _{A_{n}^{2} \rightarrow \infty} \frac{\log \log A_{n}^{2}}{\log \left(\log \left(A_{n}^{2}\right)+k\right)} \\
& \stackrel{L^{\prime} H o p i t a l}{=} \lim _{A_{n}^{2} \rightarrow \infty} \frac{\frac{1}{\log A_{n}^{2}} \frac{1}{A_{n}^{2}}}{\frac{1}{\log \left(A_{n}^{2}\right)+k} \frac{1}{A_{n}^{2}}} \\
& =\lim _{A_{n}^{2} \rightarrow \infty} \frac{\log \left(A_{n}^{2}\right)+k}{\log A_{n}^{2}} \\
& \stackrel{L^{\prime} H o p i t a l}{=} \lim _{A_{n}^{2} \rightarrow \infty} \frac{\frac{1}{A_{n}^{2}}}{\frac{1}{A_{n}^{2}}}=1
\end{aligned}
$$

$\mathrm{e}$, com isso,

$$
\begin{gathered}
\limsup _{n \rightarrow \infty} \pm \frac{S_{n}-n p}{a_{n} A_{n} \sqrt{2 p(1-p) \log \log \left(A_{n}^{2} p(1-p)\right)}}=\limsup _{n \rightarrow \infty} \pm \frac{S_{n}-n p}{a_{n} A_{n} \sqrt{2 p(1-p)} \sqrt{\log \log \left(A_{n}^{2}\right)}} \\
\stackrel{\text { q.c. }}{=} 1 \\
\Longrightarrow \limsup _{n \rightarrow \infty} \pm \frac{S_{n}-n p}{a_{n} A_{n} \sqrt{\log \log \left(A_{n}^{2}\right)}} \stackrel{\text { q.c. }}{=} \sqrt{2 p(1-p)}
\end{gathered}
$$

e assim, conclui-se a prova do Teorema 10.

Uma vez enunciados e provados os teoremas presentes em James, James e Qi (2008), são enunciados os corolários 2, 3 e 4 e provados os corolários 3 e 4 .

Para enunciar o corolário 2, é preciso introduzir a seguinte definição: 
Definição 15. Uma função $f(x)$ definida em $(0, \infty)$ é dita ser regularmente variante no infinito com índice $\alpha$ se

$$
\lim _{x \rightarrow \infty} \frac{f(t x)}{f(x)}=t^{\alpha} \text { para todo } 0<t<1
$$

Notação: $f \in R V(\alpha)$.

Por conveniência defina $a_{0}=1$. Seja $a(x)=a_{\lfloor x\rfloor}$ para $x>0$, onde $\lfloor x\rfloor$ é a parte inteira de $x$. Segue o corolário 2:

Corolário 2. Assuma que $a(x) \in R V(\theta)$, onde $\theta \in\left[0, \frac{1}{2}\right)$. Então

$$
\frac{S_{n}-n p}{\sqrt{n}} \stackrel{d}{\rightarrow} N\left(0, \frac{p(1-p)}{1-2 \theta}\right)
$$

e

$$
\limsup _{n \rightarrow \infty} \pm \frac{S_{n}-n p}{\sqrt{n \log \log n}} \stackrel{q . c .}{=} \sqrt{\frac{2 p(1-p)}{1-2 \theta}}
$$

Agora, é enunciado e provado o corolário 3.

Corolário 3. Se $\lim _{n \rightarrow \infty} \theta_{n}=\theta \in\left[0, \frac{1}{2}\right)$, então

$$
\limsup _{n \rightarrow \infty} \pm \frac{S_{n}-n p}{\sqrt{n \log \log n}} \stackrel{q . c .}{=} \sqrt{\frac{2 p(1-p)}{1-2 \theta}} .
$$

Demonstração. Para provar o corolário 3 é necessário mostrar que $a(x) \in R V(\theta)$ quando $\lim _{n \rightarrow \infty} \theta_{n}=\theta$; isto é, para todo $t \in(0,1)$,

$$
\begin{aligned}
\lim _{n \rightarrow \infty} \frac{a(n t)}{a(n)}=t^{\theta} . \\
\lim _{n \rightarrow \infty} \frac{a(n t)}{a(n)}=\lim _{n \rightarrow \infty} \frac{a_{\lfloor n t\rfloor}}{a_{n}} \\
=\lim _{n \rightarrow \infty} \frac{\prod_{i=1}^{\lfloor n t} \frac{1}{\prod_{i=1}^{n-1}} \frac{i+\theta_{i}}{i}}{i} \\
=\lim _{n \rightarrow \infty} \prod_{i=\lfloor n t\rfloor}^{n-1} \frac{i}{i+\theta_{i}}
\end{aligned}
$$


e portanto, para $n$ suficientemente grande, pela suposição de que $\lim _{n \rightarrow \infty} \theta_{n}=\theta$ e pela fórmula de Stirling:

$$
\begin{aligned}
\lim _{n \rightarrow \infty} \frac{a(n t)}{a(n)} & =\frac{\Gamma(n) \Gamma(\theta+n t)}{\Gamma(n t) \Gamma(\theta+n)} \\
& =\frac{\beta(n, \theta) \Gamma(\theta+n t)}{\Gamma(n t) \Gamma(\theta)} \\
& =\frac{\beta(n, \theta)}{\beta(n t, \theta)} \\
& \sim \frac{\Gamma(\theta) n^{-\theta}}{\Gamma(\theta)(n t)^{-\theta}} \\
& =t^{\theta}
\end{aligned}
$$

Com isso, mostramos que $a(x) \in R V(\theta)$ e utilizamos o corolário 2 para finalizar a prova.

Por fim, é enunciado e provado o corolário 4.

Corolário 4. Assuma que $\theta_{n}=\theta \in\left[0, \frac{1}{2}\right]$ para todo $n \geq 1$.

(i) Se $\theta \in\left[0, \frac{1}{2}\right)$, então

$$
\limsup _{n \rightarrow \infty} \pm \frac{S_{n}-n p}{\sqrt{n \log \log n}} \stackrel{q . c .}{=} \sqrt{\frac{2 p(1-p)}{1-2 \theta}}
$$

(ii) $\operatorname{Se} \theta=\frac{1}{2}$, então

$$
\limsup _{n \rightarrow \infty} \pm \frac{S_{n}-n p}{\sqrt{n \log n \log \log \log n}} \stackrel{q . c .}{=} \sqrt{2 p(1-p)} .
$$

Demonstração. Como $\theta_{n}=\theta$ para todo $n \geq 1$, então $\lim _{n \rightarrow \infty} \theta_{n}=\theta$ e, utilizando o corolário 3, a parte (i) do corolário 4 está provada. Para provar a parte (ii), onde $\theta_{n}=\frac{1}{2}$ para todo $n \geq 1$, iremos utilizar o lema 2:

$$
\begin{aligned}
A_{n} & =\sqrt{\sum_{i=1}^{n} a_{j}^{-2}} \sim \sqrt{\sum_{i=1}^{n}\left(\frac{\frac{1}{2} \Gamma\left(\frac{1}{2}\right)}{i^{1 / 2}}\right)^{2}} \\
& =\frac{1}{2} \Gamma\left(\frac{1}{2}\right) \sqrt{\sum_{i=1}^{n} i^{-1}} \sim \frac{1}{2} \Gamma\left(\frac{1}{2}\right) \sqrt{\int_{1}^{n} i^{-1} d_{i}} \\
& =\frac{1}{2} \Gamma\left(\frac{1}{2}\right) \sqrt{\log n}
\end{aligned}
$$


Como $a_{n} \sim \frac{\sqrt{n}}{\frac{1}{2} \Gamma\left(\frac{1}{2}\right)}$, então $a_{n} A_{n} \sim \sqrt{n \log n}$. Utilizando o teorema 10, temos que:

$$
\begin{aligned}
\limsup _{n \rightarrow \infty} \pm \frac{S_{n}-n p}{a_{n} A_{n} \sqrt{\log \log A_{n}}} & \sim \limsup _{n \rightarrow \infty} \pm \frac{S_{n}-n p}{\sqrt{n \log n \log \log A_{n}}} \\
& \sim \limsup _{n \rightarrow \infty} \pm \frac{S_{n}-n p}{\sqrt{n \log n \log \log \log n}} \\
& \stackrel{\text { q.c. }}{=} \sqrt{2 p(1-p)}
\end{aligned}
$$

e com isso concluímos a prova do corolário 4

Para mais detalhes sobre o Corolário 2, consultar o artigo referência. 



\section{CONSIDERAÇÕES FINAIS}

No decorrer deste trabalho, foi realizado um detalhamento do artigo 'Limit theorems for correlated Bernoulli random variables', por James, James e Qi (2008), além de uma revisão dos conceitos em probabilidade de convergência e martingales.

Começamos com uma revisão de conceitos necessário para o entendimento e desenvolvimento desta dissertação no Capítulo 2, sendo que os conceitos de Probabilidade foram abordados na Seção 2.1, os conceitos de Convergência foram revisados na Seção 2.2 e a apresentação dos conhecimentos em Martingales significantes para a dissertação foram introduzidos na Seção 2.3.

Posteriormente, no Capítulo 3, apresentamos ao leitor dois artigos introdutórios relacionados ao tema abordado. Na Seção 3.1 é introduzido o modelo estudado nestes dois artigos de referência, juntamente com os resultados apresentados pelos autores.

Por fim, no Capítulo 4, apresentamos um detalhamento dos resultados abordados nesta dissertação. Na Seção 4.1 é apresentado uma breve introdução do modelo proposto por James, James e Qi (2008), assim como as notações necessárias para seu entendimento. Na Seção 4.2 enunciamos e demonstramos os lemas necessários para as demonstrações da Lei Forte dos Grandes Números e do Teorema Central do Limite. Nas seções 4.3, 4.4 e 4.5 enunciamos respectivamente a parte suficiente da Lei Forte dos Grandes Números, o Teorema Central do Limite e a reciprocidade da Lei Forte dos Grandes Números para o Processo de Bernoulli Correlacionado. Por fim, na seção 4.6 foi apresentada e provada a Lei do Logaritmo Iterado e foram enunciados os demais corolários referentes a ela. 

DREZNER, Z.; FARNUM, N. A generalized binomial distribution. Communications in Statistics-Theory and Methods, Taylor \& Francis, v. 22, n. 11, p. 3051-3063, 1993. Citado 7 vezes nas páginas 17, 18, 31, 32, 33, 35 e 36.

GRIMMETT, G.; STIRZAKER, D. Probability and random processes. [S.1.]: Oxford university press, 2001. Citado na página 19.

HALL, P.; HEYDE, C. C. Martingale limit theory and its application. [S.l.]: Academic press, 1980. Citado 5 vezes nas páginas 19, 25, 27, 28 e 39.

HEYDE, C. Asymptotics and criticality for a correlated bernoulli process. Australian \& New Zealand Journal of Statistics, Wiley Online Library, v. 46, n. 1, p. 53-57, 2004. Citado 4 vezes nas páginas $17,18,31$ e 36.

JAGANNATHAN, K. Probability foundations for electrical engineers. Indian Institute of Technology Madras, 2015. Notas de aula. Citado na página 19.

JAMES, B.; JAMES, K.; QI, Y. Limit theorems for correlated bernoulli random variables. Statistics \& Probability Letters, Elsevier, v. 78, n. 15, p. 2339-2345, 2008. Citado 10 vezes nas páginas $18,27,31,35,36,40,42,44,46$ e 51.

KARR, A. F. Convergence of random variables. In: Probability. [S.1.]: Springer, 1993. p. 135162. Citado na página 24.

ROSS, S. M. Introduction to probability models. 2010. Citado 2 vezes nas páginas 19 e 23.

WILLIAMS, D. Probability with martingales. [S.1.]: Cambridge university press, 1991. Citado 2 vezes nas páginas 19 e 25. 